Review

\title{
Nurse-Coordinated Blood Pressure Telemonitoring for Urban Hypertensive Patients: A Systematic Review and Meta-Analysis
}

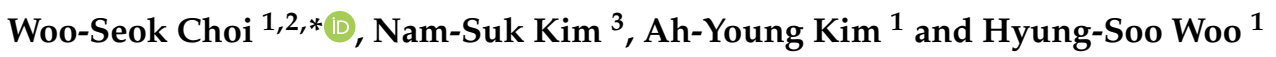 \\ 1 Moon Soul Graduate School of Future Strategy, Korea Advanced Institute of Science and Technology (KAIST), \\ Daejeon 34141, Korea; journalist@kaist.ac.kr (A.-Y.K.); h.woo@kaist.ac.kr (H.-S.W.) \\ 2 Keyu Internal Medicine Clinic, Daejeon 35250, Korea \\ 3 Public Health and Welfare Bureau, Daejeon City Hall, Daejeon 35242, Korea; heewasu@korea.kr \\ * Correspondence: keyuim@kaist.ac.kr; Tel.: +82-42-483-7554; Fax: +82-42-485-7554
}

Citation: Choi, W.-S.; Kim, N.-S.; Kim, A.-Y.; Woo, H.-S.

Nurse-Coordinated Blood Pressure Telemonitoring for Urban

Hypertensive Patients: A Systematic Review and Meta-Analysis. Int. J. Environ. Res. Public Health 2021, 18, 6892. https://doi.org/10.3390/ ijerph18136892

\section{Academic Editors:}

Irene Torres-Sánchez and Marie Carmen Valenza

Received: 29 May 2021

Accepted: 24 June 2021

Published: 27 June 2021

Publisher's Note: MDPI stays neutral with regard to jurisdictional claims in published maps and institutional affiliations.

Copyright: (c) 2021 by the authors. Licensee MDPI, Basel, Switzerland. This article is an open access article distributed under the terms and conditions of the Creative Commons Attribution (CC BY) license (https:// creativecommons.org/licenses/by/ $4.0 /)$.

\begin{abstract}
Coronavirus disease 2019 (COVID-19) has put hypertensive patients in densely populated cities at increased risk. Nurse-coordinated home blood pressure telemonitoring (NC-HBPT) may help address this. We screened studies published in English on three databases, from their inception to 30 November 2020. The effects of NC-HBPT were compared with in-person treatment. Outcomes included changes in blood pressure (BP) following the intervention and rate of BP target achievements before and during COVID-19. Of the 1916 articles identified, 27 comparisons were included in this review. In the intervention group, reductions of $5.731 \mathrm{mmHg}$ (95\% confidence interval: 4.120-7.341; $p<0.001)$ in systolic blood pressure (SBP) and $2.342 \mathrm{mmHg}(1.482-3.202 ; p<0.001)$ in diastolic blood pressure (DBP) were identified. The rate of target $\mathrm{BP}$ achievement was significant in the intervention group (risk ratio, $\mathrm{RR}=1.261,1.154-1.378 ; p<0.001$ ). The effects of intervention over time showed an SBP reduction of $3.000 \mathrm{mmHg}(-5.999-11.999)$ before 2000 and $8.755 \mathrm{mmHg}(5.177-12.334)$ in 2020. DBP reduced by $2.000 \mathrm{mmHg}(-2.724-6.724)$ before 2000 and by $3.529 \mathrm{mmHg}(1.221-5.838)$ in 2020. Analysis of the target BP ratio before $2010(R R=1.101,1.013-1.198)$ and in $2020(R R=1.906$, 1.462-2.487) suggested improved BP control during the pandemic. NC-HBPT more significantly improves office blood pressure than UC among urban hypertensive patients.
\end{abstract}

Keywords: blood pressure; hypertension; COVID-19; nurse; coordination; telemonitoring; urban

\section{Introduction}

Essential hypertension is the primary modifiable risk factor for cardiovascular disease, which is a leading cause of death according to the World Health Organization (WHO) [1]. Approximately 45\% of American adults had elevated blood pressure in the 2017-2018 time period [2,3], but only $34 \%$ were being managed to bring the blood pressure down to the recommended treatment level [4]. It has been reported that managing blood pressure to remain within the recommended level reduced the incidence of stroke by $35-40 \%$, heart attack by 20-25\%, and heart failure by more than $50 \%$ [5]. Moreover, the American Heart Association/American College of Cardiology in 2017 and the European Society of Hypertension/European Society of Cardiology in 2018 issued guidelines recommending that the target blood pressure in hypertensive patients should be regulated more strictly than recommended by previous guidelines [6,7]. Additionally, recent studies have reported that essential hypertension is one of the most common comorbidities that cause lung damage and mortality due to the coronavirus disease 2019 (COVID-19) [8,9]. Thus, strict blood pressure management is crucial to reduce the incidence of cardiovascular disease (CVD) in patients with risk factors.

Despite preventive measures such as hand washing, self-isolation, mask wearing, and social distancing, COVID-19, which first emerged in Wuhan, China, spread rapidly in high-income countries such as the United States, Spain, Germany, Italy, and Korea in the early days of the pandemic [10]. Although the number of newly confirmed patients 
has been decreasing with the administration of the vaccine since December 2020 [11], the COVID-19 pandemic is still ongoing. According to the WHO, as of April 2021, there were approximately 1.6 billion cases of severe acute respiratory syndrome coronavirus 2 (SARS-CoV-2) - the virus that causes COVID-19—infections worldwide, including 3 million deaths [12]. However, with the exception of some countries, the global vaccination rate is still too low (below 40\%) [12]. In particular, in most countries in Africa, the Middle East, and Southeast Asia, as of the end of April 2021, only approximately 10\% of the population had received at least one dose of a vaccine [12]. The drastic increase in demand for medical care during the pandemic has exposed the limitations of traditional medical systems and is adversely affecting the existing medical systems for non-communicable diseases (NCDs) such as essential hypertension [13].

During infectious disease pandemics, a high frequency of direct contact between individuals in densely populated cities can increase the infection risk even in well-established in-person medical infrastructures [14]. Thus, the need for treatment through non-face-toface interaction between doctors and patients is being emphasized more than ever before in cities [15]. Home blood pressure telemonitoring (HBPT) is widely used as an alternative measure in the management of hypertensive patients and is known to be effective in overcoming clinical inertia that may occur in face-to-face care settings [3,16-18]. In addition, remote monitoring of home blood pressure is helpful in managing patients by finding white-coat effects and masked hypertension that may be overlooked by doctors in medical institutions $[19,20]$. Studies on the effectiveness of nurse-coordinated HBPT (NC-HBPT) to prevent CVD attacks in hypertensive patients existed prior to the COVID-19 pandemic [21-25]. However, evidence on whether or not HBPT statistically enhances blood pressure control by improving communication between patients and healthcare providers has been mixed. Additionally, there is a paucity of literature indicating a solid basis for the effectiveness of nurse-led remote monitoring in the avoidance of CVD.

A coordinator plays a vital role in remote monitoring in chronic diseases [21,26], and nurses are core coordinators in the telemonitoring teams of medical institutions, working either independently or as members of a team [27]. Nurses can provide more regular follow-up, high-quality care, favorable health outcomes, and higher patient satisfaction, all equivalent to that achieved by physicians [28]. A 2005 review recommended nurse-led monitoring as an additional measure to support face-to-face therapy [29]. The literature classifies ancillary interventions to help control blood pressure into one of six categories: care led by health professionals, patient monitoring, education of medical professionals, education of patients, organizational interventions, and appointment reminder systems. Particularly, for the step-by-step care of hypertension that is not controlled by drug treatment, nurse-led regular monitoring was emphasized. However, there is uncertainty about the effectiveness of nurse-led remote monitoring in cities with established medical infrastructure and abundant medical resources. Although many studies have reported the nurses' role in HBPT intervention [3,20,28-31], no study has systematically reviewed the effectiveness of NC-HBPT in urban areas and, particularly, none have presented quantitative results according to the temporal progress. Therefore, comprehensive comparative analysis of outcome data of NC-HBPT performed in urban areas before and during the COVID-19 pandemic is important to overcome the challenges of NCD and communicable disease (CD) management and to determine the future directions for remote monitoring policies.

This study hypothesizes that NC-HBPT in urban adult hypertensive patients is not more effective than usual care (UC) in preventing CVD and that its effect over time would be identical to that of UC. To derive robust results, we conducted a systematic review and meta-analysis of randomized controlled trials (RCTs), which are placed at the top in the hierarchy of evidence-based research. Previous meta-analyses investigated the effect of HBPT in hypertensive patients [32,33], but none have integrated the results of NC-HBPT for efficient implementation in cities yet. Thus, this study aimed to examine and compare the following: mean changes in systolic blood pressure (SBP) and diastolic blood pressure 
(DBP); rates of achieving the target blood pressure after NC-HBPT; and effects of NC-HBPT over time in an urban area.

\section{Materials and Methods}

\subsection{Literature Search and Identification of Eligible Studies}

We conducted a review in accordance with the guidelines summarized in the Cochrane Handbook for Systematic Reviews of Interventions [34]. A protocol for this study has been published in the PROSPERO [CRD42020222789], which is an international prospective register of systematic reviews operated by the Center for Reviews and Dissemination at the University of York [35]. Studies evaluating HBPT that were published between the date of inception of the utilized databases and 30 November 2020, were identified. The electronic databases we used included PubMed, EBSCOhost, and the Cochrane library (CENTRAL), and the search was limited to studies published in peer-reviewed journals in English. The related search keywords included "urban", "hypertension", "remote monitoring", "telemonitoring", "telemedicine", and "randomized controlled trials." The adopted search formula was constructed by combining free terms of relevant keywords and Medical Subject Headings (MeSH) terms via truncation, Boolean operators, and phrasing. We first searched in PubMed using this structured formula and sequentially performed additional searches according to the syntax of each database (Appendix A).

All retrieved data were exported to EndNote X8.2 (Thomson Reuters, Philadelphia, PA, USA). Titles and abstracts of each study were screened, and the main text was reviewed as required. We searched for all meta-analyses conducted previously on the topic and reviewed all primary studies and relevant references in those meta-analyses. To find grey literature, we referred to related websites in the United States and Europe (e.g., OpenGrey: http:/ / opengrey.eu/ (accessed on 30 November 2020); Grey Literature Report: https: / / www.greylit.org/ (accessed on 10 December 2020). To ensure objectivity and transparency of the eligibility assessment, two of the authors (WSC and AYK) independently conducted the literature search and arrived at a mutually-agreed selection of studies.

\subsection{Inclusion and Exclusion Criteria}

An intervention group was defined as one in which patients measured their blood pressure on their own at home, reported the measurements to their doctors, and regularly visited the medical institute for follow-up, and in which a coordinator-including a nurse with or without other health care professionals-was involved in the process. As for nurses, only situations where the registered nurse monitored the patient's home blood pressure using an automatic sphygmomanometer without face-to-face contact were included. All the following actions were also included: consultation with the patient using a telephone line, mobile phone, computer, or letter; education on the disease or intervention process; execution of accompanying interventions such as a text message reminder service; and sending information regarding the patient's health status.

A control group was defined as one in which patients received routine in-person examinations at the doctor's office. Since NC-HBPT is not yet a standardized treatment itself, none of the included studies provided an equivalent of NC-HBPT, and so no active control groups were included in our analyses. Their ethnicity, level of income, and severity of hypertension were not considered separately.

We included studies (1) involving patients with essential hypertension (SBP $\geq 130 \mathrm{mmHg}$, $\mathrm{DBP} \geq 80 \mathrm{mmHg}$ ) regardless of hypertension onset or history of pharmacotherapy; (2) including patients who received treatment at an urban medical institution; (3) having an intervention that was provided for $\geq 2$ months; (4) utilizing a RCT design; and (5) involving adults aged $\geq 17$ years. The diagnostic criteria for hypertension in Europe are SBP $\geq 140$ or DBP $\geq 90$, which is higher than that in the US [7]. Thus, the diagnostic guideline for hypertension published by the 2018 ACC/AHA associations [6], which has a wider diagnostic range, was adopted in searching databases. 
However, we excluded studies (1) conducted in several regions with unclear study locations; (2) having a research location that was a mix of urban and rural areas; (3) involving patients with acute CVD or stroke with a drastic change in blood pressure; (4) involving women in the peripartum period; (5) having an intervention provided to patients in nursing homes or care facilities; (6) having an intervention provided as part of another intervention program for a different disease; (7) using a different intervention in the UC group; and (8) utilizing a cluster- or cross-over RCT design. In this study, an "urban area" was defined according to the administrative functions and population size.

\subsection{Study Selection}

A further search was conducted by reviewing the full-text manuscripts of studies identified during the first round of screening and their reference lists. All articles related to "coordinator" and "nurse" were additionally identified. Among the blinded RCTs that regularly verified the effects of these two groups, those that reported changes in blood pressure measurements before and after interventions were selected for data synthesis.

Two of the authors (NSK and HW) independently classified and excluded duplicate studies and those that did not meet the inclusion criteria. The main body of potentially valid studies was reviewed, and any disagreement between the two authors was resolved by a senior author (WSC).

\subsection{Outcomes}

The primary outcome was the weighted mean difference (WMD) of office SBP and DBP between the baseline and follow-up in the NC-HBPT and UC groups. The secondary outcome was the rate of target blood pressure achievement.

\subsection{Data Extraction and Coding}

Two researchers (NSK and AYK) independently extracted data from the selected primary studies. The data were coded in an electronic sheet using Comprehensive MetaAnalysis Software Version 2.2.064 (CMA, Biostat, Englewood, NJ, USA). Patients' age and sex, duration of remote monitoring (months), accompanying interventions, intervention pathway, the coordinator's profession, and outcome data were extracted. When a single article had different follow-up periods for the intervention or different sample sizes, or compared two or more interventions $[21,22,26,27,36-40]$, the results of each intervention were classified as independent comparative studies. For missing or inaccurate data in the primary research materials [27], we referred to journal websites or public trial registries (e.g., the US National Institutes of Health ongoing trials register) or directly contacted the authors. In cases where the standard deviation (SD) values for the mean changes were not presented [38], the values were imputed by calculating the mean of individual studies included in the review, and a sensitivity test was conducted to check for bias $[40,41]$. For the one study that did not provide baseline data [36], the data from the first assessment and the last follow-up were compared. In case of any disagreement between the researchers, a third researcher (WSC) adjudicated.

\subsection{Quality Assessment}

Two researchers (NSK and HW) independently assessed the risk of bias using Review Manager (RevMan, Version 5.4, Copenhagen: The Nordic Cochrane Center) by the Cochrane Collaboration [42], evaluating the RCTs in terms of selection, performance, detection, attrition, reporting biases, and other bias domains. A senior researcher (WCS) resolved any disagreement between the two researchers.

\subsection{Quantitative Data Synthesis}

Two researchers (WSC and NSK) analyzed the coded data. A random effects model (REM) was adopted because most of the primary studies were performed in different research institutions or by different researchers. 
For computational options for data synthesis, WMD was set as a continuous variable and relative risk (RR) as a dichotomous variable; the changes in the mean office SBP and DBP of the NC-HBPT and UC groups before and after the intervention were extracted first. Hedges' $\mathrm{g}(\mathrm{g})$ was additionally calculated to determine the appropriateness of the effect size. If the $g$-value converted from the WMD was at least 0.5 , the effect size was deemed appropriate for analysis [43]. RR was used to determine the rate of achieving the target blood pressure and was calculated using the number of samples that reached the target blood pressure during each follow-up period. All results are reported with $95 \%$ confidence intervals (CIs), and CMA software was used for statistical analysis.

Overall statistics, including weighted values, were analyzed by combining independent data. A $\chi^{2}$ test was used to assess differences between studies, and a $p$-value $<0.10$ was deemed statistically significant. Clinical heterogeneity was assessed using the $\mathrm{Q}$ statistic, tau-square $\left(\tau^{2}\right)$, and $\mathrm{I}^{2}$ statistic. Q statistic and $\tau^{2}$ interpreted the numerical values, and $\mathrm{I}^{2}$ was considered significant at $\geq 50 \%$ [43,44]. To determine the bias in each study, examine its effect on the distribution of summary effect size, and detect outliers, a sensitivity analysis using the "one study removed" method was conducted [45]. In addition, a cumulative meta-analysis was performed to identify the chronological patterns of the effect size of each study [41] (Appendix B).

After determining the effect size of each of the primary studies, their temporal changes were analyzed, and the results before and during the COVID-19 pandemic were compared.

\section{Results}

\subsection{Study Characteristics}

In total, 1916 potentially relevant articles were initially identified from databases, reference lists of each retrieved paper, and other electronic sources (Figure 1). Six datasets were additionally acquired directly from the authors, and 423 duplicates were excluded. The titles and abstracts of 1499 references were screened, and 1101 references were excluded because they were deemed inappropriate for analysis. The full copies of the remaining 398 potentially eligible articles were scrutinized. Of these, 382 studies that did not meet the aforementioned inclusion criteria-that is, studies with participants less than 17 years of age $(n=19)$, no obtainable data $(n=183)$, no nurse-coordinated intervention $(n=86)$, cluster or cross-over RCTs $(n=12)$, patients with life-threatening CVDs or stroke $(n=25)$, studies not performed in an urban setting $(n=43)$, and studies that were part of a research program for another disease $(n=14)$-were excluded. A total of 27 individual comparisons met the inclusion criteria and were selected as the final material for data synthesis.

In the meta-analysis, the mean length of NC-HBPT was 7.26 months, and the mean age of participants was 61.35 years in the intervention group and 61.62 years in the control group. Of the 27 comparisons, 20 were nurse alone-led cases [21-23,26,27,39-41,46-49], and seven cases additionally involved experts from other fields $[24,25,27,36,38]$. The characteristics of these studies are summarized in Table 1.

The medium of administering the intervention was mobile phones for 2 cases [39], mobile-web for 5 cases [38,40,47], web-based for 5 cases [21,25,37], telephone for 14 cases [22-24,26,27,36,46,49], and telephone-linked computers for 1 case [48]. A city was considered metropolitan if its population was at least one million; 11 cases were in metropolitan cities [21-23,36,38,39,47,48], and 16 were in smaller cities. 


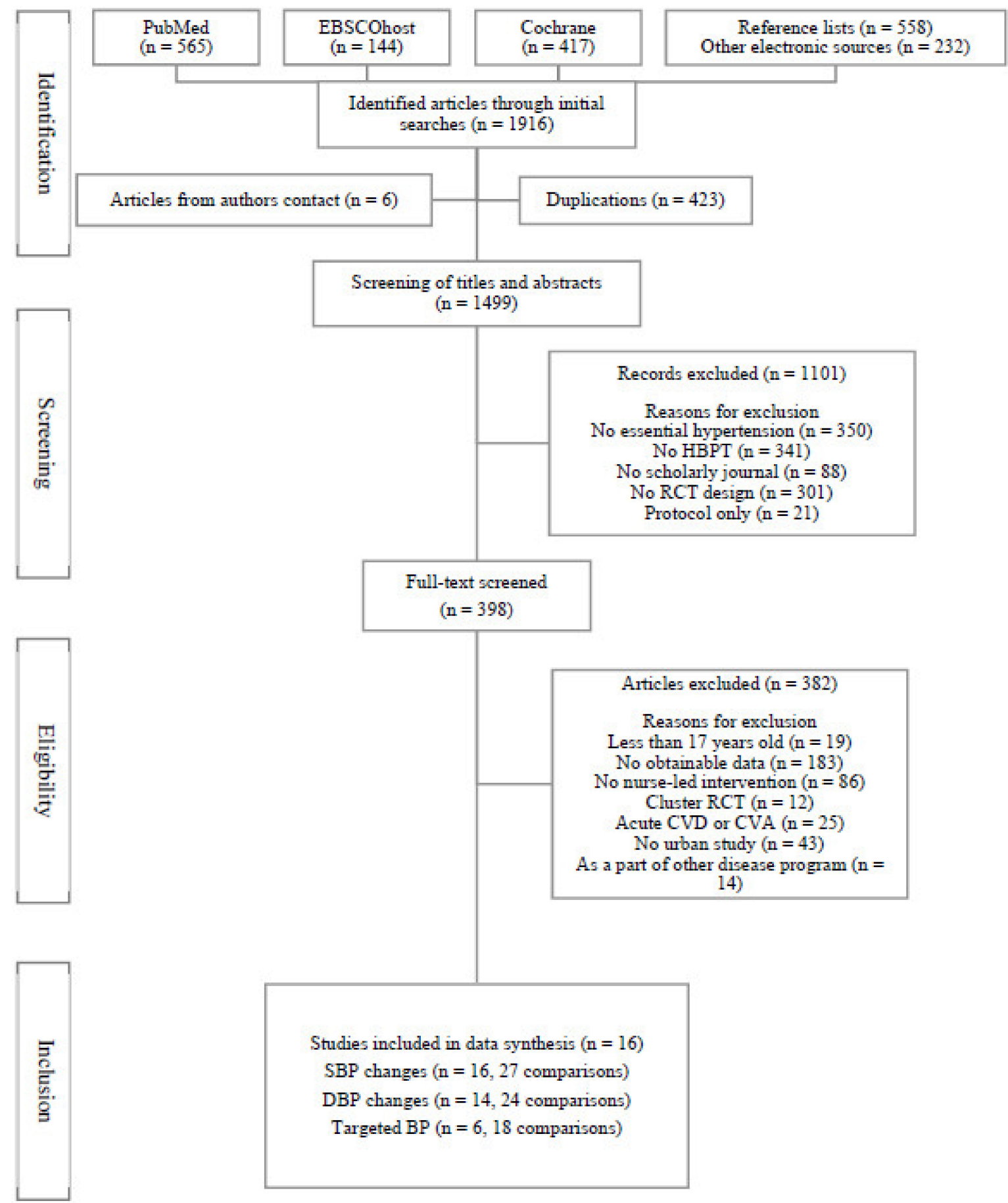

Figure 1. PRISMA flow diagram of the selection of studies included in the systematic review. Note. HBPT, home blood pressure telemonitoring; RCT, randomized controlled trial; CVD, cardiovascular disease; CVA, cerebrovascular accident; SBP, systolic blood pressure; DBP, diastolic blood pressure; BP, blood pressure. 
Table 1. Characteristics of included individual studies.

\begin{tabular}{|c|c|c|c|c|c|c|c|c|}
\hline Study & $\begin{array}{c}\text { Number of } \\
\text { Subjects (UC vs. } \\
\text { HBPT Group) }\end{array}$ & Inclusion Criteria & $\begin{array}{l}\text { The Profession } \\
\text { of Coordinator }\end{array}$ & $\begin{array}{l}\text { Name of City } \\
\text { (Country) }\end{array}$ & $\begin{array}{c}\text { Size of Urban } \\
\text { Population } \\
(\text { Over } 1,000,000)\end{array}$ & $\begin{array}{c}\text { Description of } \\
\text { Intervention } \\
\text { Pathway }\end{array}$ & $\begin{array}{l}\text { Additional } \\
\text { Support }\end{array}$ & $\begin{array}{l}\text { Main Outcome } \\
\text { Measures }\end{array}$ \\
\hline $\begin{array}{l}\text { Artinian } \\
\text { 2007A [26] }\end{array}$ & $193 / 194$ & $\begin{array}{l}\text { African-American } \\
\text { hypertensive patients }\end{array}$ & $\begin{array}{l}\text { Trained } \\
\text { registered nurse }\end{array}$ & $\begin{array}{l}\text { Detroit } \\
\text { (USA) }\end{array}$ & $\begin{array}{c}\text { No } \\
(916,952 \text { in 2007) }\end{array}$ & $\begin{array}{l}\text { Telephonic } \\
\text { transmission }\end{array}$ & $\begin{array}{l}\text { Telecounseling call } \\
\text { and patient } \\
\text { education }\end{array}$ & Changes in office BP \\
\hline $\begin{array}{l}\text { Artinian } \\
\text { 2007B [26] }\end{array}$ & $157 / 164$ & $\begin{array}{l}\text { African-American } \\
\text { hypertensive patients }\end{array}$ & $\begin{array}{l}\text { Trained } \\
\text { registered nurse }\end{array}$ & $\begin{array}{l}\text { Detroit } \\
\text { (USA) }\end{array}$ & $\begin{array}{c}\text { No } \\
(916,952 \text { in 2007) }\end{array}$ & $\begin{array}{l}\text { Telephonic } \\
\text { transmission }\end{array}$ & $\begin{array}{l}\text { Telecounseling call } \\
\text { and patient } \\
\text { education }\end{array}$ & Changes in office BP \\
\hline $\begin{array}{l}\text { Artinian } \\
\text { 2007C [26] }\end{array}$ & $169 / 167$ & $\begin{array}{l}\text { African-American } \\
\text { hypertensive patients }\end{array}$ & $\begin{array}{l}\text { Trained } \\
\text { registered nurse }\end{array}$ & $\begin{array}{l}\text { Detroit } \\
\text { (USA) }\end{array}$ & $\begin{array}{c}\text { No } \\
(916,952 \text { in 2007) }\end{array}$ & $\begin{array}{l}\text { Telephonic } \\
\text { transmission }\end{array}$ & $\begin{array}{l}\text { Telecounseling call } \\
\text { and patient } \\
\text { education }\end{array}$ & Office BP changes \\
\hline $\begin{array}{l}\text { Bosworth } \\
\text { 2007A [27] }\end{array}$ & $150 / 150$ & $\begin{array}{l}\text { Hypertensive patients } \\
\text { who are using a } \\
\text { BP-lowering medication } \\
\text { with poor (inadequate) } \\
\text { BP control }\end{array}$ & $\begin{array}{l}\text { Intervention } \\
\text { nurses }\end{array}$ & $\begin{array}{l}\text { Durham } \\
\text { (USA) }\end{array}$ & $\begin{array}{c}\text { No } \\
(217,847 \text { in 2007) }\end{array}$ & $\begin{array}{c}\text { Home BP } \\
\text { monitoring + } \\
\text { Behavioral } \\
\text { intervention }\end{array}$ & $\begin{array}{l}\text { Tailored } \\
\text { behavioral } \\
\text { intervention }\end{array}$ & $\begin{array}{l}\text { Improved rates of BP } \\
\text { control }\end{array}$ \\
\hline $\begin{array}{l}\text { Bosworth } \\
\text { 2007C [27] }\end{array}$ & $150 / 150$ & $\begin{array}{l}\text { Hypertensive patients } \\
\text { who are using a } \\
\text { BP-lowering medication } \\
\text { with poor (inadequate) } \\
\text { BP control }\end{array}$ & $\begin{array}{l}\text { Intervention } \\
\text { nurses }\end{array}$ & $\begin{array}{l}\text { Durham } \\
\text { (USA) }\end{array}$ & $\begin{array}{c}\text { No } \\
(217,847 \text { in 2007) }\end{array}$ & $\begin{array}{c}\text { Home BP } \\
\text { monitoring + } \\
\text { Combined } \\
\text { intervention }\end{array}$ & $\begin{array}{l}\text { Combined } \\
\text { behavioral and } \\
\text { medication } \\
\text { management }\end{array}$ & $\begin{array}{l}\text { Improved rates of BP } \\
\text { control }\end{array}$ \\
\hline $\begin{array}{l}\text { Bosworth } \\
2011[46]\end{array}$ & $137 / 127$ & $\begin{array}{l}\text { Treated hypertensive } \\
\text { patients }\end{array}$ & Registered nurse & $\begin{array}{l}\text { Durham } \\
\text { (USA) }\end{array}$ & $\begin{array}{c}\text { No } \\
(228,354 \text { in 2010) }\end{array}$ & $\begin{array}{l}\text { Telephonic } \\
\text { transmission }\end{array}$ & $\begin{array}{l}\text { Behavioral } \\
\text { management }\end{array}$ & $\begin{array}{l}\text { Change in BP control, } \\
\text { SBP, and DBP }\end{array}$ \\
\hline
\end{tabular}


Table 1. Cont

\begin{tabular}{|c|c|c|c|c|c|c|c|c|}
\hline Study & $\begin{array}{c}\text { Number of } \\
\text { Subjects (UC vs. } \\
\text { HBPT Group) }\end{array}$ & Inclusion Criteria & $\begin{array}{l}\text { The Profession } \\
\text { of Coordinator }\end{array}$ & $\begin{array}{l}\text { Name of City } \\
\text { (Country) }\end{array}$ & $\begin{array}{c}\text { Size of Urban } \\
\text { Population } \\
\text { (Over 1,000,000) }\end{array}$ & $\begin{array}{c}\text { Description of } \\
\text { Intervention } \\
\text { Pathway }\end{array}$ & $\begin{array}{l}\text { Additional } \\
\text { Support }\end{array}$ & $\begin{array}{l}\text { Main Outcome } \\
\text { Measures }\end{array}$ \\
\hline $\begin{array}{c}\text { Cicolini } \\
\text { 2013A [21] }\end{array}$ & $98 / 100$ & $\begin{array}{l}\text { Treated and untreated } \\
\text { hypertensive patients }\end{array}$ & Registered nurse & $\begin{array}{l}\text { Chieti } \\
\text { (Italy) }\end{array}$ & $\begin{array}{c}\text { No } \\
(51,484 \text { in 2011) }\end{array}$ & Web-based & $\begin{array}{l}\text { Email reminder } \\
\text { and phone call }\end{array}$ & $\begin{array}{l}\text { 1. Changes in BP2. } \\
\text { BMI, alcohol } \\
\text { consumption, } \\
\text { cigarette smoking, } \\
\text { adherence to therapy }\end{array}$ \\
\hline $\begin{array}{l}\text { Cicolini } \\
\text { 2013B [21] }\end{array}$ & $98 / 100$ & $\begin{array}{l}\text { Treated and untreated } \\
\text { hypertensive patients }\end{array}$ & Registered nurse & $\begin{array}{l}\text { Chieti } \\
\text { (Italy) }\end{array}$ & $\begin{array}{c}\text { No } \\
(51,484 \text { in 2011) }\end{array}$ & Web-based & $\begin{array}{l}\text { Email reminder } \\
\text { and phone call }\end{array}$ & $\begin{array}{l}\text { 1. Changes in BP2. } \\
\text { BMI, alcohol } \\
\text { consumption, } \\
\text { cigarette smoking, } \\
\text { adherence to therapy }\end{array}$ \\
\hline $\begin{array}{c}\text { Hebert } \\
\text { 2011A [23] }\end{array}$ & $83 / 85$ & $\begin{array}{l}\text { Uncontrolled } \\
\text { hypertensive patients }\end{array}$ & Registered nurse & $\begin{array}{l}\text { New York } \\
\text { (USA) }\end{array}$ & $\begin{array}{c}\text { Yes } \\
(8,174,959 \text { in } 2010)\end{array}$ & $\begin{array}{l}\text { Telephonic } \\
\text { transmission }\end{array}$ & $\begin{array}{l}\text { Information on the } \\
\text { use of home BP } \\
\text { monitor }\end{array}$ & $\mathrm{BP}$ reduction \\
\hline Hill 1999 [24] & $77 / 78$ & $\begin{array}{l}\text { Black or } \\
\text { African-American } \\
\text { hypertensive young } \\
\text { male resident within the } \\
\text { Johns-Hopkins Hospital } \\
\text { catchment area }\end{array}$ & $\begin{array}{c}\text { Nurse- } \\
\text { community } \\
\text { health worker } \\
\text { team (registered } \\
\text { nurse and health } \\
\text { worker team) }\end{array}$ & $\begin{array}{l}\text { Baltimore } \\
\text { (USA) }\end{array}$ & $\begin{array}{c}\text { No } \\
\text { (763.014 in 1990) }\end{array}$ & $\begin{array}{l}\text { Telephonic } \\
\text { transmission }\end{array}$ & $\begin{array}{l}\text { Individualized } \\
\text { counseling, } \\
\text { monthly telephone } \\
\text { call, and a home } \\
\text { visit (educational- } \\
\text { behavioral } \\
\text { intervention) }\end{array}$ & Changes in office BP \\
\hline $\begin{array}{c}\text { Kerry } \\
\text { 2012A [36] }\end{array}$ & $169 / 168$ & $\begin{array}{l}\text { Hypertensive patients } \\
\text { with history of stroke or } \\
\text { transient } \\
\text { ischemic attack }\end{array}$ & Nurse & $\begin{array}{l}\text { London } \\
\text { (UK) }\end{array}$ & $\begin{array}{c}\text { Yes } \\
(8,135,667 \text { in 2011) }\end{array}$ & $\begin{array}{l}\text { Telephonic } \\
\text { transmission }\end{array}$ & $\begin{array}{l}\text { Nurse-led } \\
\text { telephone support }\end{array}$ & Reduction of SBP \\
\hline $\begin{array}{c}\text { Kerry } \\
\text { 2012B [36] }\end{array}$ & $169 / 168$ & $\begin{array}{l}\text { Hypertensive patients } \\
\text { with history of stroke or } \\
\text { transient } \\
\text { ischemic attack }\end{array}$ & Nurse & $\begin{array}{l}\text { London } \\
\text { (UK) }\end{array}$ & $\begin{array}{c}\text { Yes } \\
(8,135,667 \text { in 2011) }\end{array}$ & $\begin{array}{l}\text { Telephonic } \\
\text { transmission }\end{array}$ & $\begin{array}{l}\text { Nurse-led } \\
\text { telephone support }\end{array}$ & Reduction of SBP \\
\hline
\end{tabular}


Table 1. Cont.

\begin{tabular}{|c|c|c|c|c|c|c|c|c|}
\hline Study & $\begin{array}{c}\text { Number of } \\
\text { Subjects (UC vs. } \\
\text { HBPT Group) }\end{array}$ & Inclusion Criteria & $\begin{array}{l}\text { The Profession } \\
\text { of Coordinator }\end{array}$ & $\begin{array}{l}\text { Name of City } \\
\text { (Country) }\end{array}$ & $\begin{array}{l}\text { Size of Urban } \\
\text { Population } \\
(\text { Over } 1,000,000)\end{array}$ & $\begin{array}{l}\text { Description of } \\
\text { Intervention } \\
\text { Pathway }\end{array}$ & $\begin{array}{l}\text { Additional } \\
\text { Support }\end{array}$ & $\begin{array}{l}\text { Main Outcome } \\
\text { Measures }\end{array}$ \\
\hline $\begin{array}{c}\text { Kim KB } \\
\text { 2014A [37] }\end{array}$ & 192/191 & $\begin{array}{l}\text { Uncontrolled } \\
\text { Korean-American } \\
\text { hypertensive seniors }\end{array}$ & $\begin{array}{l}\text { Bilingual RNs } \\
\text { and nutritionist }\end{array}$ & $\begin{array}{l}\text { Ellicott City } \\
\text { (USA) }\end{array}$ & $\begin{array}{c}\text { No } \\
(68,507 \text { in 2014) }\end{array}$ & Web-based & $\begin{array}{l}2 \mathrm{~h} \text { weekly } \\
\text { education and } \\
\text { training for } \\
6 \text { weeks and } \\
\text { monthly telephone } \\
\text { counseling }\end{array}$ & $\begin{array}{l}\text { Changes in SBP } \\
\text { and DBP }\end{array}$ \\
\hline $\begin{array}{l}\text { Kim KB } \\
\text { 2014B [37] }\end{array}$ & $187 / 185$ & $\begin{array}{l}\text { Uncontrolled } \\
\text { Korean-American } \\
\text { hypertensive seniors }\end{array}$ & $\begin{array}{l}\text { Bilingual RNs } \\
\text { and nutritionist }\end{array}$ & $\begin{array}{l}\text { Ellicott City } \\
\text { (USA) }\end{array}$ & $\begin{array}{c}\text { No } \\
(68,507 \text { in 2014) }\end{array}$ & Web-based & $\begin{array}{l}2 \mathrm{~h} \text { weekly } \\
\text { education and } \\
\text { training for } \\
6 \text { weeks and } \\
\text { monthly telephone } \\
\text { counseling }\end{array}$ & $\begin{array}{l}\text { Changes in SBP } \\
\text { and DBP }\end{array}$ \\
\hline $\begin{array}{c}\text { McMahon } \\
2005[25]\end{array}$ & $35 / 37$ & $\begin{array}{l}\text { Poorly controlled } \\
\text { diabetics and } \\
\text { hypertensive patients }\end{array}$ & $\begin{array}{c}\text { Advanced } \\
\text { practice nurse } \\
\text { and certified } \\
\text { diabetes educator }\end{array}$ & $\begin{array}{l}\text { Boston } \\
\text { (USA) }\end{array}$ & $\begin{array}{c}\text { No } \\
(559,034 \text { in 2005) }\end{array}$ & Web-based & $\begin{array}{l}\text { Telephone to } \\
\text { encourage } \\
\text { website usage }\end{array}$ & $\begin{array}{l}\text { Changes in } \mathrm{HbA}_{1 \mathrm{c}}, \mathrm{BP} \text {, } \\
\text { lipid profiles }\end{array}$ \\
\hline $\begin{array}{l}\text { Mohsen } \\
\text { 2019A [22] }\end{array}$ & $50 / 50$ & $\begin{array}{l}\text { Hypertensive patients } \\
\text { who are on } \\
\text { antihypertensive } \\
\text { medication }\end{array}$ & Staff nurse & $\begin{array}{l}\text { Shibin El Kom } \\
\quad(\text { Egypt })\end{array}$ & $\begin{array}{c}\text { No } \\
(249,611 \text { in 2018) }\end{array}$ & Telenursing & $\begin{array}{l}\text { Follow-up } \\
\text { phone calls }\end{array}$ & $\begin{array}{l}\text { Reducing arterial } \\
\text { blood pressure and } \\
\text { patients' } \\
\text { anthropometric } \\
\text { measurement }\end{array}$ \\
\hline $\begin{array}{l}\text { Mohsen } \\
\text { 2019B [22] }\end{array}$ & $50 / 50$ & $\begin{array}{l}\text { Hypertensive patients } \\
\text { who are on } \\
\text { antihypertensive } \\
\text { medication }\end{array}$ & Staff nurse & $\begin{array}{l}\text { Shibin El Kom } \\
\quad(\text { Egypt })\end{array}$ & $\begin{array}{c}\text { No } \\
(249,611 \text { in 2018) }\end{array}$ & Telenursing & $\begin{array}{l}\text { Follow-up } \\
\text { phone calls }\end{array}$ & $\begin{array}{l}\text { Reducing arterial } \\
\text { blood pressure and } \\
\text { patients' } \\
\text { anthropometric } \\
\text { measurement }\end{array}$ \\
\hline
\end{tabular}


Table 1. Cont.

\begin{tabular}{|c|c|c|c|c|c|c|c|c|}
\hline Study & $\begin{array}{c}\text { Number of } \\
\text { Subjects (UC vs. } \\
\text { HBPT Group) }\end{array}$ & Inclusion Criteria & $\begin{array}{l}\text { The Profession } \\
\text { of Coordinator }\end{array}$ & $\begin{array}{l}\text { Name of City } \\
\text { (Country) }\end{array}$ & $\begin{array}{l}\text { Size of Urban } \\
\text { Population } \\
\text { (Over } 1,000,000)\end{array}$ & $\begin{array}{l}\text { Description of } \\
\text { Intervention } \\
\text { Pathway }\end{array}$ & $\begin{array}{l}\text { Additional } \\
\text { Support }\end{array}$ & $\begin{array}{l}\text { Main Outcome } \\
\text { Measures }\end{array}$ \\
\hline $\begin{array}{c}\text { Pan } \\
\text { 2018A [38] }\end{array}$ & $55 / 52$ & $\begin{array}{l}\text { Hypertensive patients } \\
\text { with uncontrolled BP }\end{array}$ & $\begin{array}{l}\text { GP, a hyperten- } \\
\text { sionspecialist, a } \\
\text { general nurse, an } \\
\text { information } \\
\text { manager }\end{array}$ & $\begin{array}{l}\text { Beijing } \\
\text { (China) }\end{array}$ & $\begin{array}{c}\text { Yes } \\
(19,612,368 \text { in } \\
2010)\end{array}$ & $\begin{array}{l}\text { Smartphone } \\
\text { application }\end{array}$ & $\begin{array}{l}\text { Follow-up phone } \\
\text { calls }\end{array}$ & $\begin{array}{l}\text { The reduction in } \\
\text { systolic blood } \\
\text { pressure }\end{array}$ \\
\hline $\begin{array}{c}\text { Pan } \\
\text { 2018B [38] }\end{array}$ & $55 / 52$ & $\begin{array}{l}\text { Hypertensive patients } \\
\text { with uncontrolled BP }\end{array}$ & $\begin{array}{l}\text { GP, a hyperten- } \\
\text { sionspecialist, a } \\
\text { general nurse, an } \\
\text { information } \\
\text { manager }\end{array}$ & $\begin{array}{l}\text { Beijing } \\
\text { (China) }\end{array}$ & $\begin{array}{c}\text { Yes } \\
(19,612,368 \text { in } \\
2010)\end{array}$ & $\begin{array}{l}\text { Smartphone } \\
\text { application }\end{array}$ & $\begin{array}{l}\text { Follow-up phone } \\
\text { calls }\end{array}$ & $\begin{array}{l}\text { The reduction in } \\
\text { systolic blood } \\
\text { pressure }\end{array}$ \\
\hline $\begin{array}{l}\text { Park MJ } \\
2009[47]\end{array}$ & $21 / 28$ & $\begin{array}{l}\text { Obese hypertensive } \\
\text { patients }\end{array}$ & Registered nurse & $\begin{array}{c}\text { Seoul } \\
\text { (S. Korea) }\end{array}$ & $\begin{array}{c}\text { Yes } \\
(10,208,302 \text { in } \\
2009)\end{array}$ & $\begin{array}{l}\text { Mobile or internet } \\
\text { transmission }\end{array}$ & $\begin{array}{l}\text { Short message } \\
\text { service by cellular } \\
\text { phone and } \\
\text { internet } \\
\text { recommendation }\end{array}$ & $\begin{array}{l}\text { Changes in BP, body } \\
\text { weight, waist } \\
\text { circumference, and } \\
\text { serum lipid profile }\end{array}$ \\
\hline $\begin{array}{l}\text { Rahmani } \\
\text { Pour } \\
\text { 2019A [39] }\end{array}$ & $21 / 21$ & $\begin{array}{l}\text { Hypertensive patients, } \\
\text { use of antihypertensive } \\
\text { medications }\end{array}$ & $\begin{array}{l}\text { Instructors of a } \\
\text { faculty of nursing } \\
\text { and midwifery } \\
\text { and several } \\
\text { cardiologists }\end{array}$ & $\begin{array}{l}\text { Tehran } \\
\text { (Iran) }\end{array}$ & $\begin{array}{c}\text { Yes } \\
(8,693,706 \text { in 2016) }\end{array}$ & Non-ISMS & Follow-up & $\begin{array}{l}\text { No significant } \\
\text { differences among the } \\
\text { groups with respect to } \\
\text { the baseline SBP } \\
\text { and DBP }\end{array}$ \\
\hline $\begin{array}{c}\text { Shea } \\
2006[48]\end{array}$ & $347 / 333$ & $\begin{array}{l}\text { Diabetic hypertensive } \\
\text { patients }\end{array}$ & $\begin{array}{l}\text { Nurse case } \\
\text { manager }\end{array}$ & $\begin{array}{l}\text { New York } \\
\text { (USA) }\end{array}$ & $\begin{array}{c}\text { Yes } \\
(8,143,197 \text { in } 2005)\end{array}$ & $\begin{array}{l}\text { Telephone-linked } \\
\text { web-enabled } \\
\text { computer system }\end{array}$ & $\begin{array}{l}\text { Web-based } \\
\text { messaging }\end{array}$ & $\begin{array}{l}\text { Changes in } \mathrm{HbA}_{1 \mathrm{c}}, \mathrm{BP} \text {, } \\
\text { and cholesterol level }\end{array}$ \\
\hline
\end{tabular}


Table 1. Cont.

\begin{tabular}{|c|c|c|c|c|c|c|c|c|}
\hline Study & $\begin{array}{c}\text { Number of } \\
\text { Subjects (UC vs. } \\
\text { HBPT Group) }\end{array}$ & Inclusion Criteria & $\begin{array}{l}\text { The Profession } \\
\text { of Coordinator }\end{array}$ & $\begin{array}{l}\text { Name of City } \\
\text { (Country) }\end{array}$ & $\begin{array}{l}\text { Size of Urban } \\
\text { Population } \\
\text { (Over 1,000,000) }\end{array}$ & $\begin{array}{l}\text { Description of } \\
\text { Intervention } \\
\text { Pathway }\end{array}$ & $\begin{array}{l}\text { Additional } \\
\text { Support }\end{array}$ & $\begin{array}{l}\text { Main Outcome } \\
\text { Measures }\end{array}$ \\
\hline $\begin{array}{l}\text { Wakefield } \\
2011[49]\end{array}$ & $97 / 83$ & $\begin{array}{l}\text { Type } 2 \text { diabetics and } \\
\text { hypertensive patients }\end{array}$ & Registered nurse & $\begin{array}{l}\text { Iowa City } \\
\text { (USA) }\end{array}$ & $\begin{array}{c}\text { No } \\
(68,036 \text { in 2010) }\end{array}$ & $\begin{array}{l}\text { Telephonic } \\
\text { transmission }\end{array}$ & $\begin{array}{l}\text { Telephonic } \\
\text { transmission and } \\
\text { nurse care } \\
\text { management }\end{array}$ & $\begin{array}{l}\text { Changes in } \mathrm{HbA}_{1 \mathrm{c}} \\
\text { and SBP }\end{array}$ \\
\hline $\begin{array}{c}\text { Zha } \\
\text { 2020A [40] }\end{array}$ & $13 / 12$ & $\begin{array}{l}\text { Underserved and } \\
\text { vulnerable urban } \\
\text { population on HTN } \\
\text { medication with } \\
\text { diagnosed uncontrolled } \\
\text { hypertension }\end{array}$ & $\begin{array}{l}\text { Two nurses in the } \\
\text { community } \\
\text { health center }\end{array}$ & $\begin{array}{l}\text { Newark } \\
\text { (USA) }\end{array}$ & $\begin{array}{c}\text { No } \\
(281,764 \text { in 2016) }\end{array}$ & $\begin{array}{l}\text { Mobile Health } \\
\text { (Smart phone } \\
\text { or tablet) }\end{array}$ & $\begin{array}{l}\text { Three training } \\
\text { sessions }\end{array}$ & $\begin{array}{l}\text { No significant change } \\
\text { in systolic BP, the } \\
\text { potential to facilitate } \\
\text { hypertension } \\
\text { management }\end{array}$ \\
\hline $\begin{array}{l}\text { Zha } \\
\text { 2020B [40] }\end{array}$ & $13 / 12$ & $\begin{array}{l}\text { Underserved and } \\
\text { vulnerable urban } \\
\text { population on HTN } \\
\text { medication with } \\
\text { diagnosed uncontrolled } \\
\text { hypertension }\end{array}$ & $\begin{array}{l}\text { Two nurses in the } \\
\text { community } \\
\text { health center }\end{array}$ & $\begin{array}{l}\text { Newark } \\
\text { (USA) }\end{array}$ & $\begin{array}{c}\text { No } \\
(281,764 \text { in 2016) }\end{array}$ & $\begin{array}{l}\text { Mobile Health } \\
\text { (Smart phone } \\
\text { or tablet) }\end{array}$ & $\begin{array}{l}\text { Three training } \\
\text { sessions }\end{array}$ & $\begin{array}{l}\text { No significant change } \\
\text { in systolic BP, the } \\
\text { potential to facilitate } \\
\text { hypertension } \\
\text { management }\end{array}$ \\
\hline
\end{tabular}

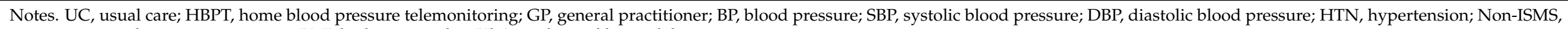
non-interactive short message service; BMI, body mass index; HbA1c, glycated hemoglobin. 


\subsection{Assessment of Risk of Bias}

The selection and performance processes for most trials were appropriate. In the case of attrition or reporting bias domain, there were studies with an unclear or high risk of bias. A primary study at a low risk of bias in at least four domains was deemed to be of high quality [50], and 23 comparisons were identified as having a high risk of bias in fewer than four domains, suggesting that the overall quality of the materials was relatively high.

Publication bias was assessed using the trim-and-fill method [51]. The point estimates of SBP based on a funnel plot were as follows: WMD $=5.327 \mathrm{mmHg}$ and $g=0.723$ $(0.445-1.002, p<0.001)$; there were no trimmed studies (Figure 2). For DBP, $g=0.362$ $(0.222-0.503, p<0.001)$, which represented a meaningful effect size (Figure 3$)$. The funnel plots of both SBP and DBP showed good visual symmetry, and there were no imputed studies for SBP and DBP. Thus, it was concluded that the potential publication biases did not affect the primary outcomes of the materials included in this study.

\section{Funnel Plot of Standard Error by Hedges's g}

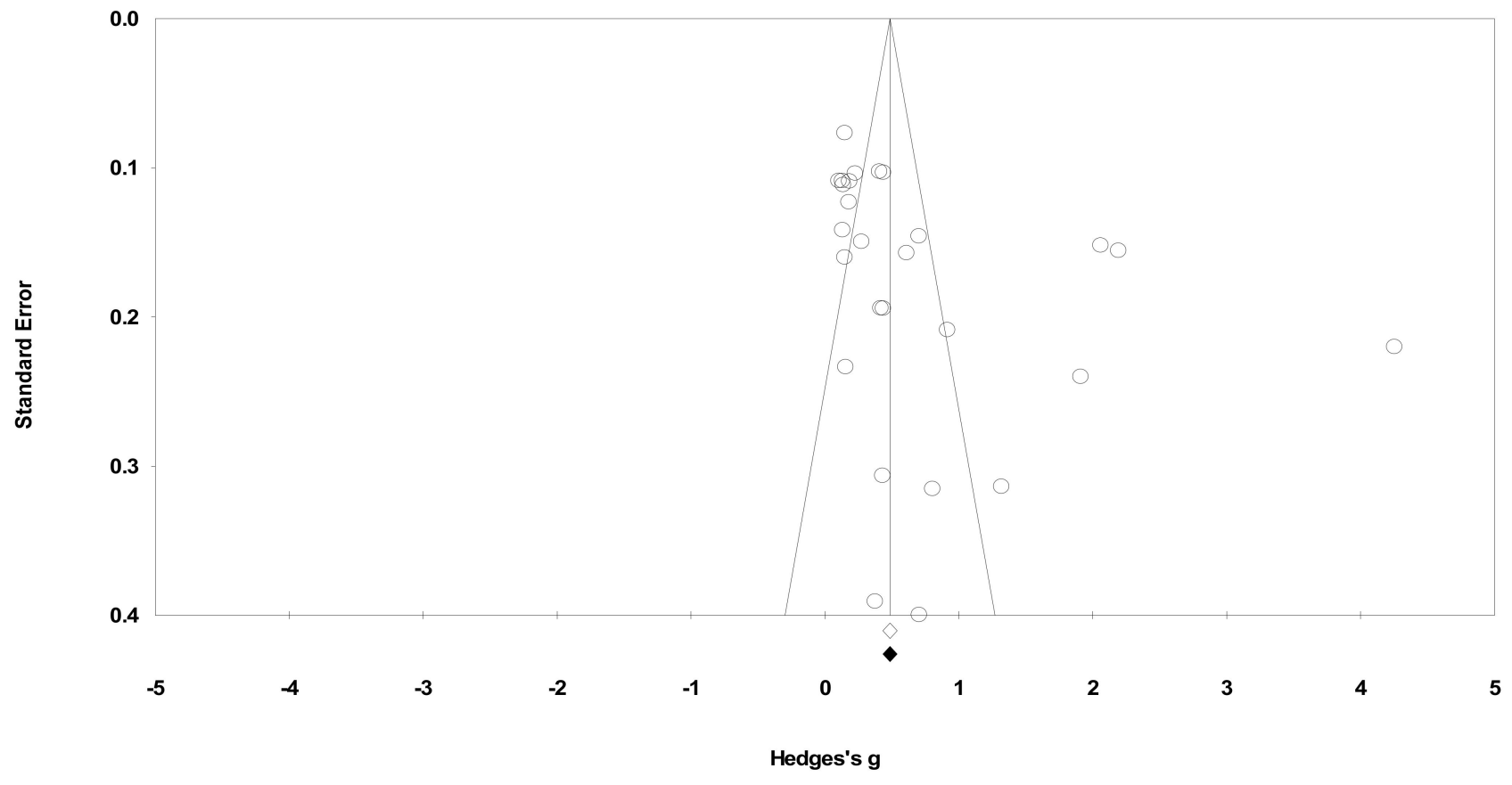

Figure 2. Funnel plot of systolic blood pressure by Hedges' g (plot observed and imputed), random effects model. Notes. Summary effect size $(\diamond)$; Summary effect size of imputed studies $(\bullet)$; Individual studies $(\bigcirc)$.

Publication bias was also assessed for the rate of reaching the target office blood pressure as a secondary outcome. The funnel plot showed good visual symmetry (Figure 4). One study was imputed, but the difference in point estimates was not significant (observed RR, 1.261 , vs. adjusted RR, 1.240). Thus, the publication bias by the potentially unpublished study did not affect the RR effect size. 


\section{Funnel Plot of Standard Error by Hedges's $\mathbf{g}$}

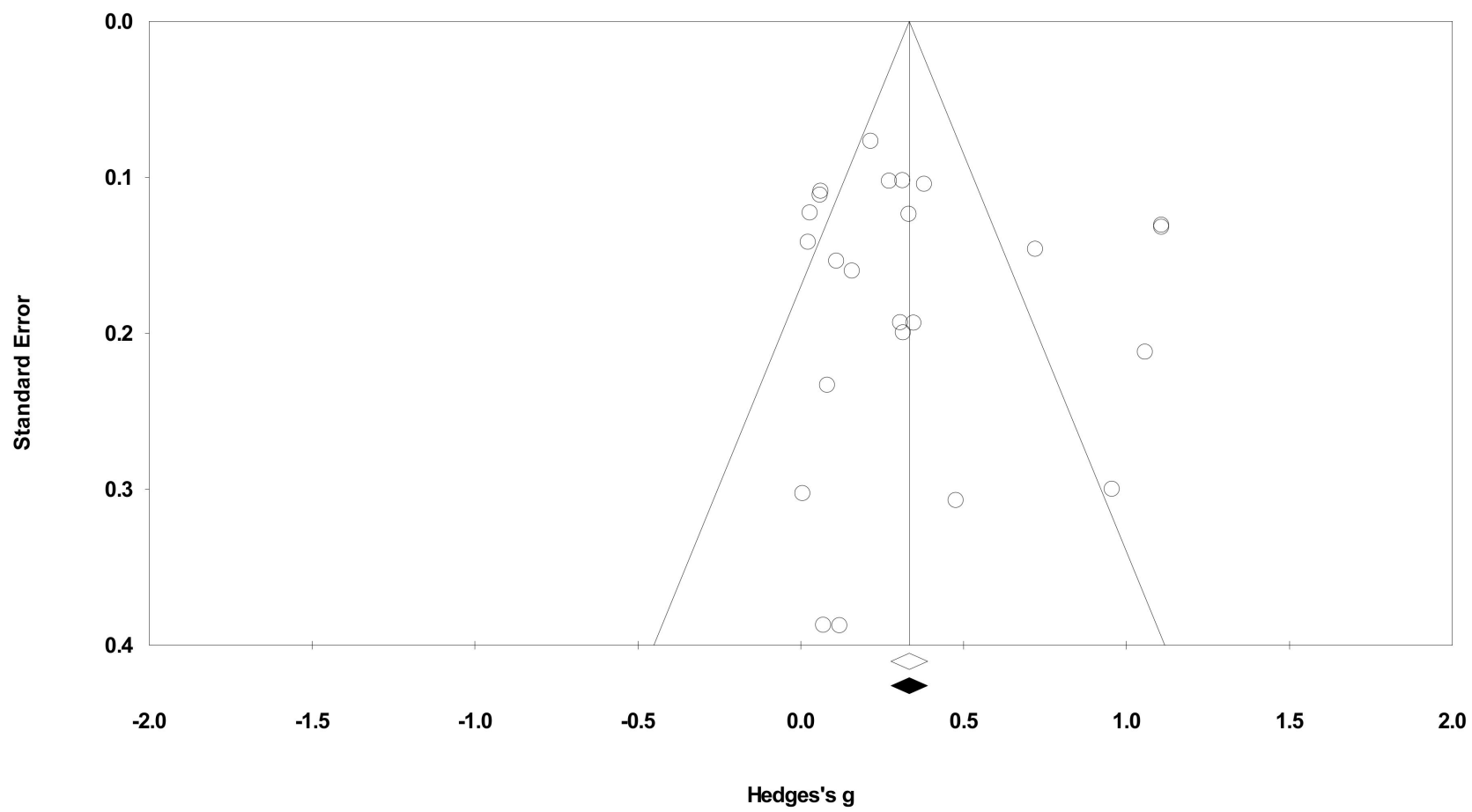

Figure 3. Funnel plot of diastolic blood pressure by Hedges' g (plot observed and imputed), random effects model. Notes. Summary effect size $(\diamond)$; Summary effect size of imputed studies $(\bullet)$; Individual studies $(\bigcirc)$.

\section{Funnel Plot of Standard Error by Log risk ratio}

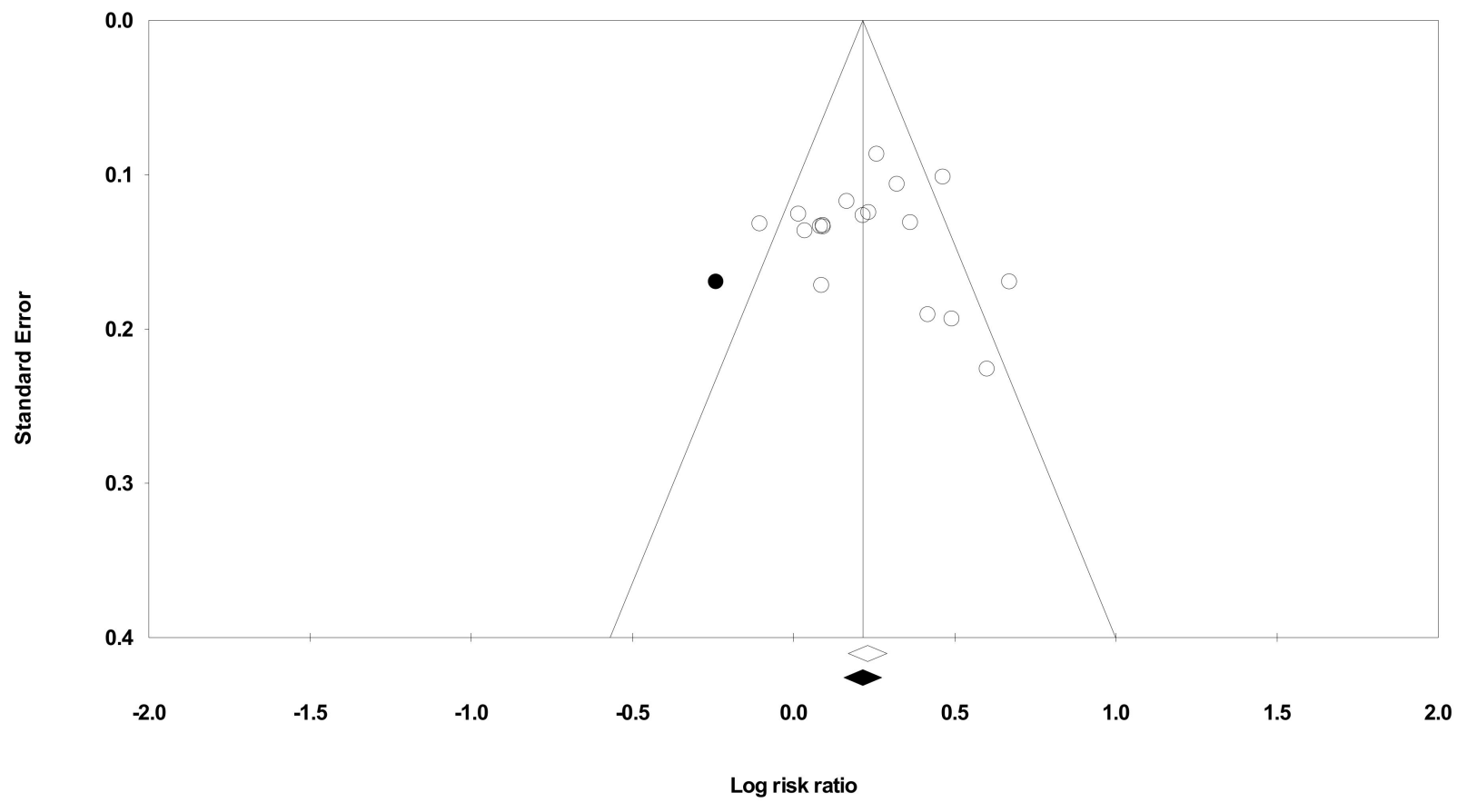

Figure 4. Funnel plot of rate of target blood pressure by log risk ratio (plot observed and imputed), random effects model. Notes. Summary effect size $(\diamond)$; Imputed study $(\bullet)$; Summary effect size of imputed studies $(\bullet)$; Individual studies $(\bigcirc)$. 


\subsection{Primary Outcomes}

\subsubsection{SBP Changes by Nurse-Coordinated HBPT}

Data were pooled from the 27 comparisons (16 studies) that included 2860 patients in the NC-HBPT group and 2918 patients in the control group, comprising a total of 5778 patients in both groups [21-27,36-40,46-49]. A significant reduction in blood pressure was observed in the nurse-led intervention group compared with the UC group $(5.731 \mathrm{mmHg}$; 4.120-7.341; $p<0.001$; Figure 5), and heterogeneity was significant among the studies $\left(\mathrm{I}^{2}=71.717 \% ; p<0.001\right)$. In the sensitivity test [45], the individual studies did not affect the summary point estimates (WMD: $3.822-7.578 \mathrm{mmHg}$ ).

Author (year) (ref.) Outcome

Statistics for each study

Difference Lower Upper

in means limit limit $p$-Value

\begin{tabular}{llrrrl|} 
Artinian,2007A & SBP & 8.100 & 4.190 & 12.010 & 0.000 \\
Artinian,2007B & SBP & 2.900 & -1.547 & 7.347 & 0.201 \\
Artinian,2007C & SBP & 4.000 & -0.413 & 8.413 & 0.076 \\
Bosworth,2007A & SBP & 3.100 & -1.074 & 7.274 & 0.146 \\
Bosworth,2007B & SBP & 6.400 & 2.009 & 10.791 & 0.004 \\
Bosworth,2007C & SBP & 3.300 & -1.125 & 7.725 & 0.144 \\
Bosworth,2011 & SBP & 3.100 & -1.158 & 7.358 & 0.154 \\
Cicolini,2013A & SBP & 1.000 & -1.827 & 3.827 & 0.488 \\
Cicolini,2013B & SBP & 5.000 & 2.180 & 7.820 & 0.001 \\
Hebert,2011A & SBP & 13.100 & 7.394 & 18.806 & 0.000 \\
Hill,1999 & SBP & 3.000 & -2.889 & 8.889 & 0.318 \\
Kerry,2013A & SBP & 2.100 & -2.284 & 6.484 & 0.348 \\
Kerry,2013B & SBP & 2.700 & -1.737 & 7.137 & 0.233 \\
Kim KB,2014A & SBP & 8.000 & 4.251 & 11.749 & 0.000 \\
Kim KB,2014B & SBP & 4.000 & 0.262 & 7.738 & 0.036 \\
McMahon,2005 & SBP & 3.000 & -6.200 & 12.200 & 0.523 \\
Mohsen,2020A & SBP & 9.400 & 5.184 & 13.616 & 0.000 \\
Mohsen,2020B & SBP & 20.000 & 15.723 & 24.277 & 0.000 \\
Pan,2018A & SBP & 6.900 & 1.745 & 12.055 & 0.009 \\
Pan,2018B & SBP & 6.600 & 1.445 & 11.755 & 0.012 \\
Park MJ,2009 & SBP & 11.900 & 6.839 & 16.961 & 0.000 \\
Rahmani Pour,2020A SBP & 6.670 & -3.070 & 16.410 & 0.180 \\
Rahmani Pour,2020B SBP & 11.720 & 2.203 & 21.237 & 0.016 \\
Shea,2006 & SBP & 3.190 & -0.213 & 6.593 & 0.066 \\
Wakefield,2011 & SBP & 4.770 & -0.243 & 9.783 & 0.062 \\
Zha,2020A & SBP & 2.170 & -1.862 & 6.202 & 0.292 \\
Zha,2020B & SBP & 3.600 & -0.115 & 7.315 & 0.058 \\
& & 5.731 & 4.120 & 7.341 & 0.000
\end{tabular}

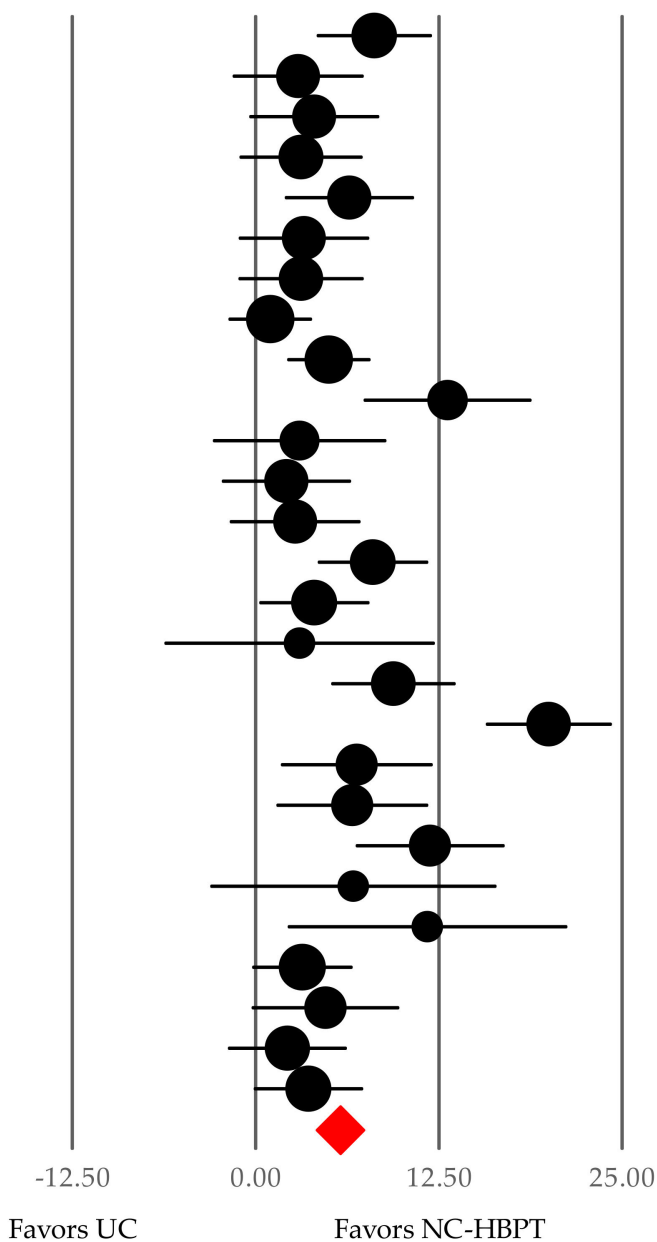

Figure 5. Difference in means of office systolic blood pressure changes by nurse-coordinated intervention. Notes. Point estimate of individual study $(\bullet)$; Summary effect size $(\bullet)$; SBP, systolic blood pressure; UC, usual care; NC-HBPT, nursecoordinated home blood pressure telemonitoring.

Changes in WMD over time were examined across four time frames. The SBP reduction during time frame I (inception to 2000) was $3.000 \mathrm{mmHg}(-5.999$ to 11.999; $p=0.514)$ [24]. SBP reductions of $5.150 \mathrm{mmHg}(2.383-7.898 ; p<0.001)$ and $4.990 \mathrm{mmHg}$ $(2.565-7.415 ; p<0.001)$ were observed in time frames II (2001-2010) $[25-27,47,48]$ and III (2011-2019) $[21,23,33-35,43,46]$, respectively (Figure 6). A significant SBP reduction of $8.755 \mathrm{mmHg}(5.177-12.334 ; p<0.001)$ was observed in time frame IV $(2020)[22,39,40]$, the year in which the COVID-19 pandemic began. 


\begin{tabular}{ll} 
Group by & Author (year) (ref.) \\
\cline { 1 - 1 } Time Interval & \\
& \\
I & Hill,1999 \\
I & \\
II & Artinian,2007A \\
II & McMahon,2005 \\
II & Park M,2009 \\
II & Artinian,2007B \\
II & Shea,2006 \\
II & Artinian,2007C \\
II & Bosworth,2007A \\
II & Bosworth,2007B \\
II & Bosworth,2007C \\
II & \\
III & Hebert,2011A \\
III & Kim KB,2014A \\
III & Kim KB,2014B \\
III & Pan,2018A \\
III & Pan,2018B \\
III & Kerry,2013A \\
III & Kerry,2013B \\
III & Wakefield,2011 \\
III & Bosworth,2011 \\
III & Gicolini,2013A \\
III & Gicolini,2013B \\
III & \\
IV & Mohsen,2020A \\
IV & Mohsen,2020B \\
IV & Rahmani Pour,2020A \\
IV & Rahmani Pour,2020B \\
IV & Zha,2020A \\
IV & Zha,2020B \\
IV & \\
Overall & \\
\hline & \\
\hline
\end{tabular}

\begin{tabular}{|c|c|c|c|c|}
\hline \multirow[t]{2}{*}{ Outcome } & \multicolumn{4}{|c|}{ Statistics for each study } \\
\hline & $\begin{array}{c}\text { Difference } \\
\text { in means }\end{array}$ & $\begin{array}{c}\text { Lower } \\
\text { limit }\end{array}$ & $\begin{array}{c}\text { Upper } \\
\text { limit }\end{array}$ & $\mathrm{p}$-Value \\
\hline \multirow[t]{2}{*}{ SBP } & 3.000 & -2.889 & 8.889 & 0.318 \\
\hline & 3.000 & -5.999 & 11.999 & 0.514 \\
\hline SBP & 8.100 & 4.190 & 12.010 & 0.000 \\
\hline SBP & 3.000 & -6.200 & 12.200 & 0.523 \\
\hline SBP & 11.900 & 6.839 & 16.961 & 0.000 \\
\hline SBP & 2.900 & -1.547 & 7.347 & 0.201 \\
\hline SBP & 3.190 & -0.213 & 6.593 & 0.066 \\
\hline SBP & 4.000 & -0.413 & 8.413 & 0.076 \\
\hline SBP & 3.100 & -1.074 & 7.274 & 0.146 \\
\hline SBP & 6.400 & 2.009 & 10.791 & 0.004 \\
\hline \multirow[t]{2}{*}{ SBP } & 3.300 & -1.125 & 7.725 & 0.144 \\
\hline & 5.140 & 2.383 & 7.898 & 0.000 \\
\hline SBP & 13.100 & 7.394 & 18.806 & 0.000 \\
\hline SBP & 8.000 & 4.251 & 11.749 & 0.000 \\
\hline SBP & 4.000 & 0.262 & 7.738 & 0.036 \\
\hline SBP & 6.900 & 1.745 & 12.055 & 0.009 \\
\hline SBP & 6.600 & 1.445 & 11.755 & 0.012 \\
\hline SBP & 2.100 & -2.284 & 6.484 & 0.348 \\
\hline SBP & 2.700 & -1.737 & 7.137 & 0.233 \\
\hline SBP & 4.770 & -0.243 & 9.783 & 0.062 \\
\hline SBP & 3.100 & -1.158 & 7.358 & 0.154 \\
\hline SBP & 1.000 & -1.827 & 3.827 & 0.488 \\
\hline \multirow[t]{2}{*}{ SBP } & 5.000 & 2.180 & 7.820 & 0.001 \\
\hline & 4.990 & 2.565 & 7.415 & 0.000 \\
\hline SBP & 9.400 & 5.184 & 13.616 & 0.000 \\
\hline SBP & 20.000 & 15.723 & 24.277 & 0.000 \\
\hline SBP & 6.670 & -3.070 & 16.410 & 0.180 \\
\hline SBP & 11.720 & 2.203 & 21.237 & 0.016 \\
\hline SBP & 2.170 & -1.862 & 6.202 & 0.292 \\
\hline \multirow[t]{3}{*}{ SBP } & 3.600 & -0.115 & 7.315 & 0.058 \\
\hline & 8.755 & 5.177 & 12.334 & 0.000 \\
\hline & 5.860 & 3.455 & 8.266 & 0.000 \\
\hline
\end{tabular}

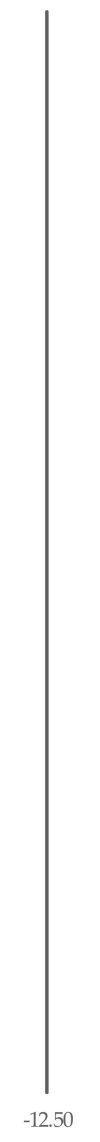

Favors UC

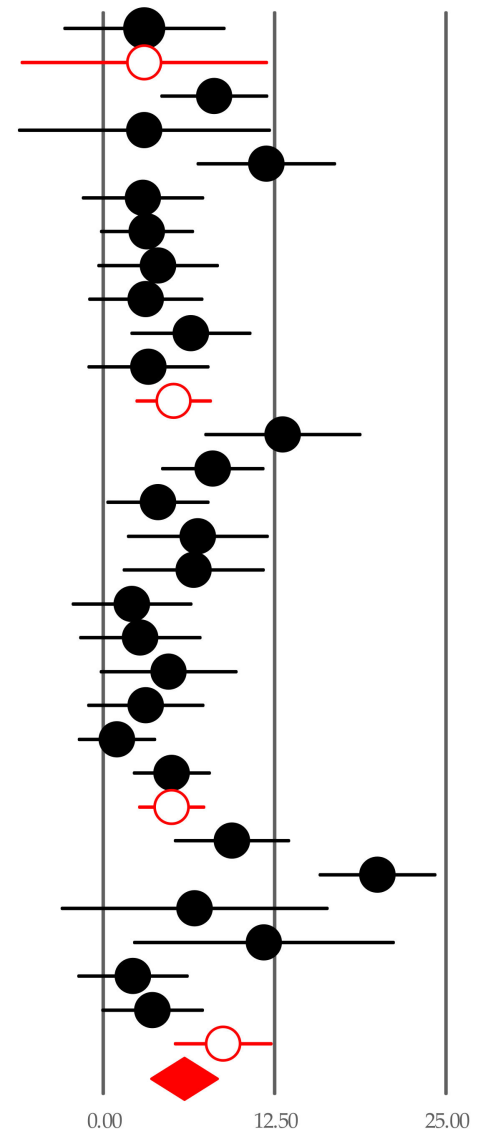

Favors NC-HBPT

Figure 6. Difference in means of office systolic blood pressure changes over time. Notes. Point estimate of individual study $(\bullet)$; Subgroup $(\circ)$; Summary effect size $(\bullet)$; DBP, diastolic blood pressure; UC, usual care; NC-HBPT, nurse-coordinated home blood pressure telemonitoring.

\subsubsection{DBP Changes by Nurse-Led Coordination}

The WMD analysis of DBP was possible using data pooled from 24 comparisons (14 studies) [21-27,37-40,46-48], and data from 4928 patients (2445 from the NC-HBPT group and 2483 from the UC group) were analyzed. There was a significant decrease in blood pressure in the intervention group compared with the control $(2.342 \mathrm{mmHg}$, 1.482-3.202; $p<0.001$; Figure 7). Significant heterogeneity was observed between the comparisons $\left(\mathrm{I}^{2}=51.380 \% ; p=0.002\right)$. In the sensitivity test, individual studies did not significantly alter the summary effect size (WMD: $1.343-3.359 \mathrm{mmHg}$ ). 
Author (year) (ref.) Outcome

\begin{tabular}{l} 
Artinian,2007A \\
Artinian,2007B \\
Artinian,2007C \\
Bosworth,2007A \\
Bosworth,2007B \\
Bosworth,2007C \\
Bosworth,2011 \\
Cicolini,2013A \\
Cicolini,2013B \\
Hebert,2011A \\
Hill,1999 \\
Kim KB,2014A \\
Kim KB,2014B \\
McMahon,2005 \\
Mohsen,2020A \\
Mohsen,2020B \\
Pan,2018A \\
Pan,2018B \\
Park MJ,2009 \\
Rahmani Pour,2020A \\
Rahmani Pour,2020B \\
Shea,2006 \\
Zha,2020A \\
Zha,2020B \\
\hline A
\end{tabular}

DBP

DBP

DBP

DBP

DBP

DBP

DBP

DBP

DBP

DBP

DBP

DBP

DBP

DBP

DBP

DBP

DBP

DBP

DBP

DBP

DBP

DBP

DBP

DBP
Statistics for each study

$$
\text { Difference }
$$
in means

$\begin{array}{cc}\text { Lower } & \text { Upper } \\ \text { limit } & \text { limit }\end{array}$

4.000

0.800

0.800

0.300

1.000

1.000

0.300

0.100

3.500

1.500

2.000

3.000

4.000

1.000

2.700

8.700

3.400

3.000

7.600

3.530

0.040

2.300

1.040

0.540

2.342

$$
1.379
$$

$-2.147$

$-2.030$

$-2.755$

$-2.244$

$-2.163$

$-2.587$

$-1.114$

2.204

$-2.654$

$-1.683$

0.743

1.755

$-3.858$

$-0.816$

5.222

$-0.007$

$-0.407$

3.050

$-2.400$

$-5.745$

0.661

$-5.110$

$-5.152$

1.482

$$
6.621
$$$$
\begin{aligned}
& 6.621 \\
& 3.747
\end{aligned}
$$

3.630

3.355

4.244

4.163

3.187

1.314

4.796

5.654

5.683

5.257

6.245

5.858

6.216

12.178

6.807

6.407

12.150

9.460

5.825

3.939

7.190

6.232

3.201

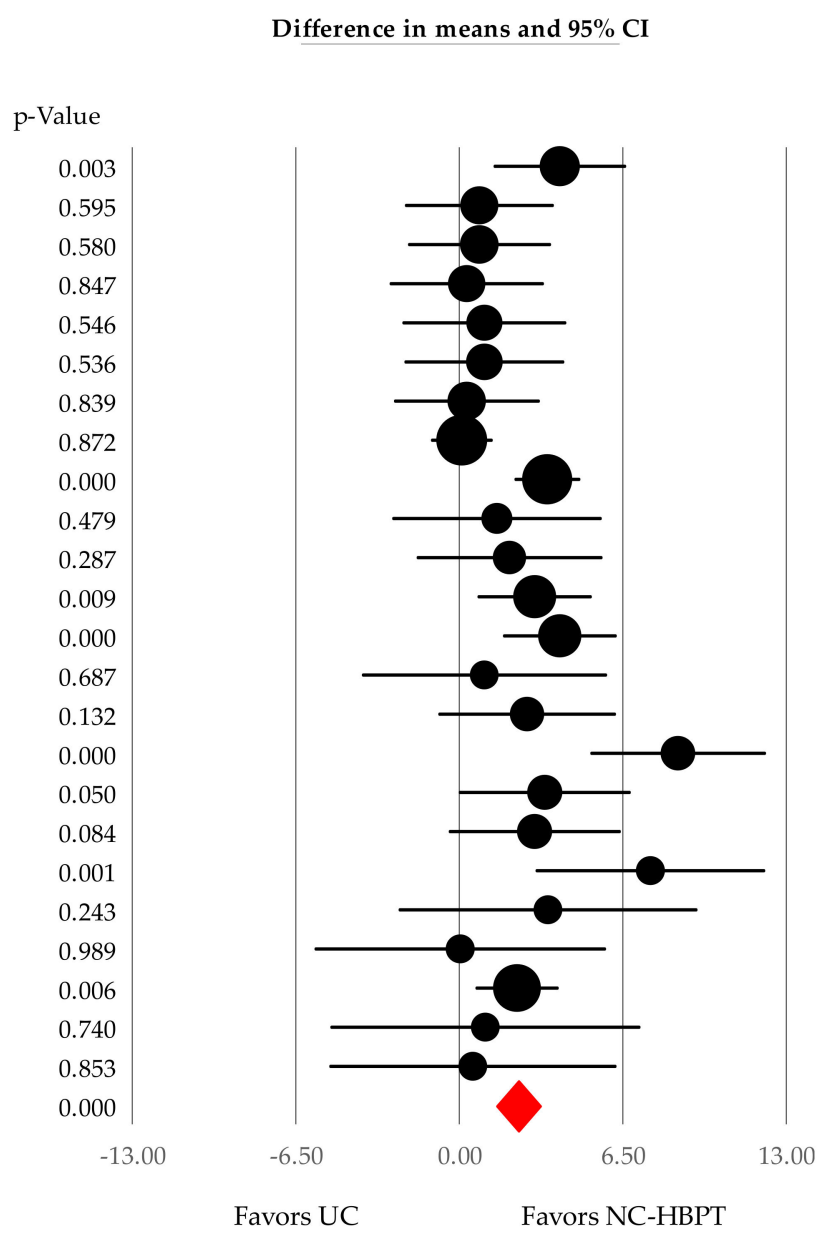

Difference in means and $95 \%$ CI

Favors UC

Figure 7. Difference in means of office diastolic blood pressure changes by nurse-coordinated intervention. Notes. Point estimate of individual study $(\bullet)$; Summary effect size $(\bullet)$; DBP, diastolic blood pressure; UC, usual care; NC-HBPT, nursecoordinated home blood pressure telemonitoring.

The WMD of DBP in the NC-HBPT group was $2.000 \mathrm{mmHg}(-2.724$ to $6.724 ; p=0.407)$ in time frame I [24], $1.947 \mathrm{mmHg}(0.524-3.370 ; p=0.007)$ in time frame II [25-27,47,48], $2.327 \mathrm{mmHg}(0.958-3.695 ; p<0.001)$ in time frame III $[21,23,37,38,46]$, and $3.529 \mathrm{mmHg}$ $(1.221-5.838 ; p=0.003)$ in time frame IV $[22,39,40]$, suggesting that the effect of the intervention was statistically greater closer to the COVID-19 outbreak period (Figure 8). 


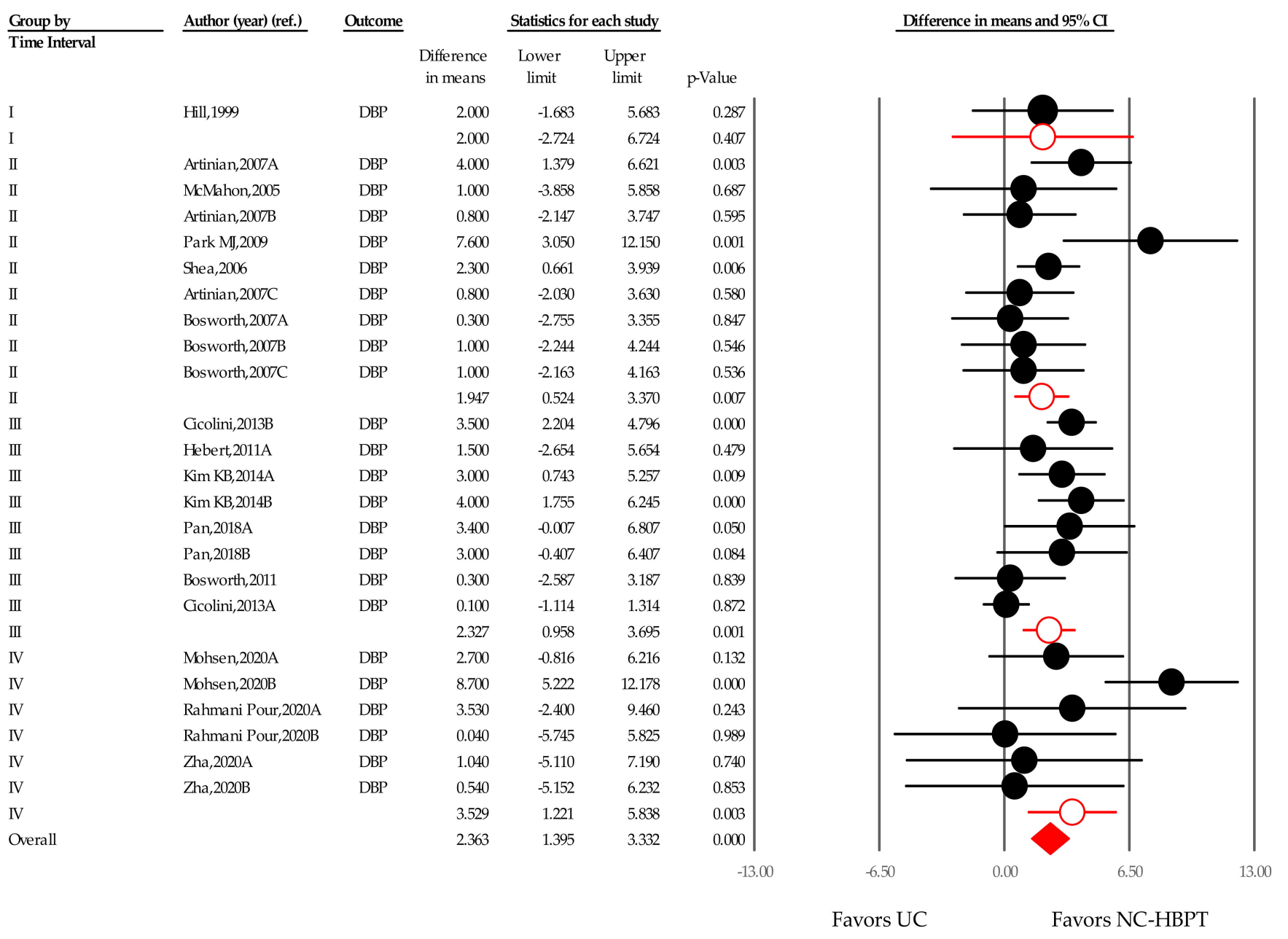

Figure 8. Difference in means of office diastolic blood pressure changes over time. Notes. Summary effect size $(\bullet)$; Point estimate of individual study $(\bullet)$; Subgroup (०); UC, usual care; NC-HBPT, nurse-coordinated home blood pressure telemonitoring.

\subsection{Secondary Outcomes}

Rate of Reaching the Target Office Blood Pressure

Using 18 available comparisons (6 studies) [21-23,27,37,38], we calculated the rate of reaching the target office blood pressure in the NC-HBPT group. Data from 4078 patients (2021 from the intervention group and 2057 from the UC group) showed that the rate of reaching the target office blood pressure was significantly higher in the intervention group than in the UC group (RR 1.261, 1.154-1.378; $p<0.001$ ). Heterogeneity between the studies was substantial $\left(\mathrm{I}^{2}=51.6 \%\right)$. In the sensitivity test, study removal did not have a significant effect on the summary effect size ( $p<0.001$; range: 1.133-1.399).

Changes in the rate of reaching the target blood pressure over time were analyzed by combining the data obtained after the year 2000. RR values of $1.101(1.013-1.198 ; p=0.024)$, $1.400(1.279-1.534 ; p<0.001)$, and $1.906(1.462-2.487 ; p<0.001)$ were found for time frames II [27], III [21,23,37,38], and IV [22], respectively, showing a clear improvement rate.

\subsection{Subgroup Analysis \\ 3.5.1. Size of City}

Cities were classified based on their population size, where small- to medium-sized cities had fewer than 1 million residents, and large cities had more than 1 million residents. In small to medium cities $(\mathrm{n}=16$; participants $=3713)[18,21,24-27,37,40,49]$, the decrease in SBP by NC-HBPT was $4.134 \mathrm{mmHg}\left(2.275-5.992, p<0.001 ; \mathrm{I}^{2}=7.934\right)$, while SBP 
decreased by $8.355 \mathrm{mmHg}\left(5.937-10.773, p<0.001 ; \mathrm{I}^{2}=82.533\right)$ in large cities $(\mathrm{n}=11$; participants $=2069)$, compared with the UC group $[22,23,36,38,39,47,48]$ (Appendix C).

\subsubsection{Setting}

When groups were classified according to the setting in which the study was conducted, the reduction in SBP due to NC-HBPT in primary care clinics $(n=5$; participants = 1741) was $3.793 \mathrm{mmHg}\left(0.450-7.136, p=0.026 ; \mathrm{I}^{2}=0.000\right)[27,46,48]$, $4.353 \mathrm{mmHg}\left(1.877-6.829, p=0.001 ; \mathrm{I}^{2}=34.921\right)$ in community health centers $(\mathrm{n}=9$; participants $=1704)[21,26,38,40]$, and $7.781 \mathrm{mmHg}$ of SBP $\left(1.375-5.483, p<0.001 ; \mathrm{I}^{2}=79.627\right)$ in hospitals $(\mathrm{n}=13$; participants $=2337)$ compared with the UC group $[22-25,36,37,39,47,49]$.

\subsubsection{Duration}

For the full duration of NC-HBPT, a total of 5278 people (NC-HBPT group $=2612 \mathrm{vs}$. $\mathrm{UC}=2666$ ) were analyzed. The WMD of SBP was consistently decreased throughout the intervention, by $6.694 \mathrm{mmHg}$ after 3 months $\left(3.644-9.744, p<0.001 ; \mathrm{I}^{2}=72.511\right)[21,22,26,38-40,47]$, $6.608 \mathrm{mmHg}$ after 6 months (3.777-9.444, $\left.p<0.001 ; \mathrm{I}^{2}=85.761\right)[21,22,26,37-39,41,50]$, and $3.573 \mathrm{mmHg}$ at 12 months of intervention $\left(0.796-6.349, p=0.012 ; \mathrm{I}^{2}=12.877\right)$ compared with the UC group $[24,25,27,36,37,46,48]$.

\subsubsection{Coordinator's Profession}

A total of 4398 patients were included in the analysis to derive the effectiveness of the nurse-only interventions $(\mathrm{n}=20)$. The decrease in WMD of SBP by nurse alone was $6.132 \mathrm{mmHg}\left(4.262-8.002, p<0.001 ; \mathrm{I}^{2}=78.074\right)[21,22,26,27,37,39,40,46-49]$. Cases where nurses collaborated with other professionals $(n=7)$ included a total of 1384 people and showed a decrease in SBP of $4.465 \mathrm{mmHg}\left(1.122-7.807, p=0.009 ; \mathrm{I}^{2}=75.373\right)[24,25,27,36,38]$. When classified according to the professions nurses collaborated with, SBP decreased by $2.399 \mathrm{mmHg}\left(-3571-8.369, \mathrm{I}^{2}=0.000\right)$ for nutritionists, $3.000 \mathrm{mmHg}(-8.682-14.682$, $\left.\mathrm{I}^{2}=0.000\right)$ for lifestyle educators, and $3.000 \mathrm{mmHg}\left(-6.300-12.300, \mathrm{I}^{2}=34.534\right)$ for community health workers compared with UC. When the collaboration was with a doctor, SBP was reduced by $6.626 \mathrm{mmHg}\left(1.599-11.652, \mathrm{I}^{2}=67.129\right)$ compared with the control group.

\subsubsection{Medically Underserved Area}

The effect in medically underserved areas reported in each primary study (participants $=5278)$ was analyzed. In underserved areas $(n=10$; participants $=2852)$, the decrease in SBP was $5.100 \mathrm{mmHg}\left(2.484-7.717, p<0.001 ; \mathrm{I}^{2}=49.707\right)[23,24,26,37,40,48]$, whereas SBP was reduced by $6.150 \mathrm{mmHg}(4.041-8.259, p<0.001)$ in non-marginalized areas ( $\mathrm{n}=17$; participants $=2930)[21,22,25,27,36,38,39,46,47,49]$. In the latter areas, the heterogeneity was substantial $\left(\mathrm{I}^{2}=78.192\right)$.

\section{Discussion}

This study assessed the effectiveness of NC-HBPT in patients with hypertension, a common NCD, in an urban setting. If remote medical services are defined as delivering patients' biological information and managing diseases using information and communications technology (ICT) [52], NC-HBPT can be considered a safe, effective, and timely intervention in the current pandemic situation, which has drastically increased the demand for medical resources, in addition to social measures to prevent the spread of COVID19 [53].

In this meta-analysis, NC-HBPT achieved an SBP reduction of $5.731 \mathrm{mmHg}(4.120-7.341$, $p<0.001)$ at an average of 7.26 months. A large-scale meta-analysis using individual patient data showed that an SBP reduction of $4 \mathrm{mmHg}$ can reduce the CVD incidence to 10 events / 1000 cases in hypertensive patients with a moderate 5-year CVD risk (11-15\%) [54]. A previous meta-analysis examining the effects of HBPT in the same setting without consideration of coordinators reported an SBP reduction of $3.482 \mathrm{mmHg}$ 
(2.459-4.505, $p<0.001)$ [55]. Thus, the increased effect of NC-HPBT in preventing CVD can be considered clinically significant.

When examining the effect of NC-HBPT over time, SBP was relatively low at the beginning of the intervention but increased by $3.000 \mathrm{mmHg}(-5.999-11.999, p=0.514)$, and the maximum reduction of $8.755 \mathrm{mmHg}(5.177-12.334, p<0.001)$ was achieved in 2020, when the pandemic began. Although the number of studies included in time frame I is insufficient and lacks significance, the data cannot be completely ignored, as the aforementioned trend undeniably exists based on the comparison of time frame II values (5.140 mmHg, 2.383-7.898, $p<0.001$ ) with those in time frames III and IV. Greater SBP reduction was achieved by NC-HBTP over time. Considering the preference for non-faceto-face contact in 2020 and 2021 due to the COVID-19 pandemic and the increased demand for remote medical services, it can be extrapolated from our results that an increase in the potential effectiveness of remote medical services with nurse coordination is possible.

Based on evidence from previous literature suggesting that NC-HBPT is effective $[3,20]$, we examined the effect of a nurse-alone intervention through 20 comparisons [21-23,26, $27,37,39,40,46-49]$. HBPT coordinated by a nurse alone achieved an SBP reduction of $6.132 \mathrm{mmHg}(4.262-8.002, p<0.001)$, which was not inferior to the mean reduction of interventions by all coordinators $(5.731 \mathrm{mmHg})$. Moreover, compared with NC-HBPT additionally coordinated by an expert from another field, HBPT was even more effective when a physician was involved in the intervention $(\mathrm{n}=3 ; 6.626 \mathrm{mmHg}, 1.599-11.652)[27,38]$. HBPT showed limited effectiveness when coordinated by community health workers (3.000 $\mathrm{mmHg},-6.300-12.300)$ [24], nutritionists $(2.399 \mathrm{mmHg},-3.571-8.369)$ [36], or lifestyle educators (3.000 mmHg, $-8.682-14.682)$ [25].

We also found that NC-HBPT was associated with a larger reduction in DBP $(2.342 \mathrm{mmHg}$, $1.482-3.201, p<0.001)$ than HBPT in the same setting but without consideration of coordinators $(1.638 \mathrm{mmHg}, 1.084-2.192, p<0.001)$ [55]. Similar patterns were observed in DBP and SBP changes over time. A higher reduction in DBP of $3.529 \mathrm{mmHg}(1.221-5.838$, $p=0.003)$ was observed in time frame IV $[22,39,40]$ than in time frame II $(1.947 \mathrm{mmHg}$, $0.524-3.370 ; p=0.007)[25-27,47,48]$.

HBPT coordinated by a nurse alone achieved a DBP reduction of $2.389 \mathrm{mmHg}$ $(1.393-3.384, p<0.001)[21-24,26,27,37,39,40,47-49]$, and additional coordination by a doctor achieved a DBP reduction of $2.440 \mathrm{mmHg}(-0.166-5.047, p=0.066)$ [26,38], showing a significantly greater DBP reduction when HBPT is additionally coordinated by experts from other specific fields than in NC-HBPT [24-26,38]. Although the reason for the differences in blood pressure reductions according to the profession of the additional coordinator is unclear, it may have to do with social and organizational factors, the coordinator's level of medical knowledge and experience, and similarity in the education received by the coordinators $[56,57]$. However, since the number of cases in which NC-HBPT was coordinated by experts from other fields was small and the results were not statistically significant, further research is needed to more accurately determine the validity of the effect of the intervention.

Palmas et al. (2006) reported that a lack of awareness of the benefits of remote medical technologies and the burden of using these technologies have contributed to the low expectations for remote medical services in urban areas [58]. However, the high percentage of hypertensive patients who are highly susceptible to COVID-19 and the environmental factors that are found in densely populated cities contribute to excessive medical demands that cannot be handled by traditional medical systems [59]. Therefore, the importance of remote monitoring technology as a means to efficiently provide medical services with limited resources is being increasingly emphasized. To the best of our knowledge, this is the first meta-analysis to examine the effect of NC-HBPT on urban hypertensive patients over time. We have derived meaningful results regarding the benefits of nurse-coordinated interventions.

In this study, the heterogeneity of the summary effect sizes was found to be substantial. Specifically, the $\mathrm{I}^{2}$ for SBP and DBP were $71.717 \%(p<0.001)$ and $51.380 \%(p=0.002)$, 
respectively. Thus, the authors applied a random effects model to the analysis, which did not completely remove the heterogeneity. Therefore, subgroup analysis was performed to assess the causes of the heterogeneity, and several moderators were explored that revealed clinical implications, along with evaluation of heterogeneity.

Despite selecting well-founded blinded RCTs through a transparent and systematic process and deriving solid and integrated results for the primary outcomes without publication biases, our study has some limitations. First, an extensive literature search was conducted on reliable and relevant databases using a structured formula, but the number of studies included was low. By building a more precise search formula, the reliability of our results could be improved. Second, since each time frame did not contain an equal number of comparisons, the analysis results for different periods were not based on the same number of studies. Including as many studies as possible, with an equal number of studies per time frame, can overcome this limitation. Third, while the researchers reasonably set the duration of each time frame to 9 and 10 years according to the technological changes and historical events to explore temporal patterns of outcomes of NC-HBPT, the distinction between each time frame may not have been clear. Thus, it may be necessary to set the time frames based on the turning points of ICT development to increase the precision of the findings. Lastly, for studies that compared interventions with different coordinators or lengths of follow-up, each comparison was counted as an independent primary study, but there were cases where multiple comparisons were included in one study. Although there was substantial heterogeneity between each comparison, and no statistical errors were observed, there may be a lack of optimal scientific robustness due to the methodological limitations of a random effects model. This limitation can also be overcome by updating the results based on a larger number of studies.

\section{Conclusions}

This study revealed that NC-HBPT for urban hypertensive patients can deliver clinically and statistically good BP reduction in terms of avoiding CVD outbreak when compared with UC. Our findings may have meaningful implications for policymakers in urban areas that are planning to introduce remote monitoring systems or in areas with inefficient telemedicine systems. However, some included studies in this analysis lack quality. Thus, although the evidence for the benefit of NC-HBPT was found in this review, further research is necessary on the nurses' roles as coordinators. Additionally, future work must consider the effect of multiple variables on NC-HBPT for more efficient implementation of the intervention system in urban areas.

Author Contributions: Conceptualization, N.-S.K. and W.-S.C.; methodology, N.-S.K., A.-Y.K., H.-S.W. and W.-S.C.; software, A.-Y.K. and W.-S.C.; validation, N.-S.K., A.-Y.K., H.-S.W. and W.-S.C.; formal analysis, N.-S.K., A.-Y.K. and W.-S.C.; investigation, H.-S.W. and W.-S.C.; writing—original draft preparation, N.-S.K. and W.-S.C.; writing-review and editing, H.-S.W. and W.-S.C. All authors have read and agreed to the published version of the manuscript.

Funding: This research received no external funding.

Institutional Review Board Statement: Not applicable.

Informed Consent Statement: Not applicable.

Conflicts of Interest: The authors declare no conflict of interest.

\section{Appendix A. Searching Formula}

$(((((($ “Hypertension”[Mesh]) OR ((hypertensi*) OR high blood pressure) )) AND (((((““Urban Population”[Mesh]) OR “Urban Health”[Mesh]) OR “Urban Health Services” [Mesh]) OR "Cities"[Mesh])) OR ((((urban*) OR city) OR cities) OR central cit*)))) AND ((((("Telemedicine”[Mesh]) OR “Telemetry"[Mesh]) OR “Blood Pressure Monitoring, Ambulatory"[Mesh])) OR $\left(\left(\left(\left(()\left(\left((\right.\right.\right.\right.\right.\right.$ telemedicine $)$ OR telemetry) OR telenurs*) OR telemonitor* $\left.{ }^{*}\right)$ OR eHealth) OR telehealth) OR remote monitor*) OR technology) OR telephone) OR 
smartphone) OR internet) $)))$ AND $(((((((($ randomised controlled trial) OR randomized controlled) OR controlled clinical trial)) OR (((((((randomised[Title/Abstract]) OR randomized[Title/Abstract]) OR placebo[Title/Abstract]) OR drug therapy[Title/Abstract]) OR groups[Title/Abstract]) OR clinical trials as topic[Title/Abstract]) OR randomly[Title/ Abstract]) OR trial[Title/Abstract]))) NOT cluster randomized controlled trials)) NOT cross over study)

Appendix B. Cumulative Meta-Analysis Presenting the Summative Effect at Each Time Point

\section{Author (year) (ref.)}

\begin{tabular}{|c|c|c|c|}
\hline & & Point & $\mathrm{p}$-Value \\
\hline Hill,1999 & SBP & 3.000 & 0.318 \\
\hline McMahon,2005 & SBP & 3.000 & 0.236 \\
\hline Shea,2006 & SBP & 3.129 & 0.029 \\
\hline Artinian,2007A & SBP & 4.779 & 0.001 \\
\hline Artinian,2007B & SBP & 4.397 & 0.000 \\
\hline Artinian,2007C & SBP & 4.346 & 0.000 \\
\hline Bosworth,2007A & SBP & 4.143 & 0.000 \\
\hline Bosworth,2007B & SBP & 4.433 & 0.000 \\
\hline Bosworth,2007C & SBP & 4.306 & 0.000 \\
\hline Park MJ,2009 & SBP & 4.950 & 0.000 \\
\hline Bosworth,2011 & SBP & 4.761 & 0.000 \\
\hline Hebert,2011A & SBP & 5.340 & 0.000 \\
\hline Wakefield,2011 & SBP & 5.287 & 0.000 \\
\hline Zha,2020A & SBP & 5.026 & 0.000 \\
\hline Cicolini,2013A & SBP & 4.658 & 0.000 \\
\hline Cicolini,2013B & SBP & 4.663 & 0.000 \\
\hline Kerry,2013A & SBP & 4.504 & 0.000 \\
\hline Kerry,2013B & SBP & 4.395 & 0.000 \\
\hline Kim KB,2014A & SBP & 4.624 & 0.000 \\
\hline Kim KB,2014B & SBP & 4.578 & 0.000 \\
\hline Pan,2018A & SBP & 4.664 & 0.000 \\
\hline Pan,2018B & SBP & 4.731 & 0.000 \\
\hline Mohsen,2020A & SBP & 4.959 & 0.000 \\
\hline Mohsen,2020B & SBP & 5.692 & 0.000 \\
\hline Rahmani Pour,2020A & SBP & 5.710 & 0.000 \\
\hline Rahmani Pour,2020B & SBP & 5.831 & 0.000 \\
\hline \multirow[t]{2}{*}{ Zha,2020B } & SBP & 5.731 & 0.000 \\
\hline & & 5.731 & 0.000 \\
\hline
\end{tabular}

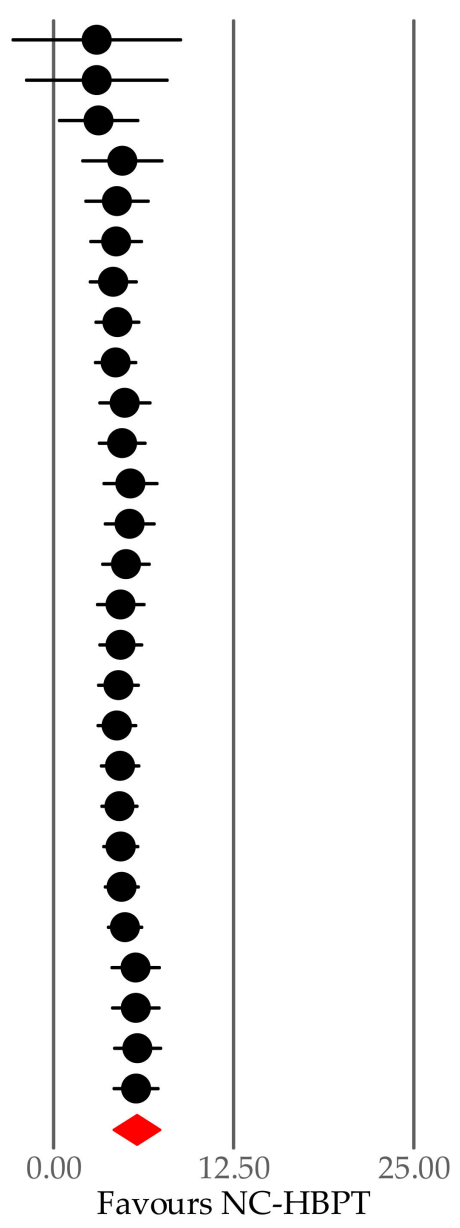

Figure A1. Cumulative meta-analysis presenting the summative effect at each time point. Note. SBP, systolic blood pressure; UC, usual care; NC-HBPT, nurse-coordinated home blood pressure telemonitoring; Summary effect size ( $\bullet$ ); Point estimate of individual study $(\bullet)$. 


\section{Appendix C. Subgroup Analysis}

Table A1. Subgroup analysis.

\begin{tabular}{|c|c|c|c|c|}
\hline Category & Number of Studies & $\begin{array}{l}\text { Summary WMD of } \\
\text { SBP, mmHg (95\% CI) }\end{array}$ & $\begin{array}{c}\text { Heterogeneity, } \mathbf{I}^{2}(\%) \\
\text { by FEM }\end{array}$ & $p$-Value of $\mathrm{I}^{2}$ \\
\hline Overall & 27 & 5731 & 71.717 & $p<0.001$ \\
\hline \multicolumn{5}{|l|}{ City size (population) } \\
\hline Under 1 million & 16 & $4.134(2.275-5.992)$ & 7.934 & $p=0.063$ \\
\hline Over 1 million & 11 & 8.355 (5.937-10.773) & 82.533 & $p<0.001$ \\
\hline \multicolumn{5}{|l|}{ Setting } \\
\hline Primary care clinic & 5 & $3.793(0.450-7.136)$ & 0.000 & $p=0.086$ \\
\hline $\begin{array}{l}\text { Community health } \\
\text { center }\end{array}$ & 9 & $4.353(1.877-6.829)$ & 34.921 & $p=0.039$ \\
\hline Hospital & 13 & 7.781 (5.483-10.079) & 79.627 & $p<0.001$ \\
\hline \multicolumn{5}{|l|}{ Duration (month) } \\
\hline 3 or less & 8 & $6.694(3.644-9.744)$ & 72.511 & $p=0.001$ \\
\hline 6 & 8 & $6.608(3.771-9.444)$ & 85.761 & $p<0.001$ \\
\hline 12 & 9 & $3.573(0.796-6.349)$ & 12.877 & $\mathrm{P}=0.042$ \\
\hline \multicolumn{5}{|l|}{$\begin{array}{l}\text { Medically underserved } \\
\text { area }\end{array}$} \\
\hline Not underserved & 17 & $6.150(4.041-8.259)$ & 78.192 & $p<0.001$ \\
\hline Underserved & 10 & $4.424(2.484-7.717)$ & 49.707 & $p=0.036$ \\
\hline \multicolumn{5}{|l|}{$\begin{array}{l}\text { Collaboration with } \\
\text { other professionals }\end{array}$} \\
\hline No (Nurse-alone) & 20 & 6.132 & 78.074 & $p<0.001$ \\
\hline Yes & 7 & 4.465 & 75.373 & $p=0.633$ \\
\hline
\end{tabular}

Note. WMD, weighted mean difference; SBP, systolic blood pressure; CI, confidence interval; FEM, fixed effect model.

\section{References}

1. WHO. Hypertension. Available online: https://www.who.int/news-room/fact-sheets/detail/hypertension (accessed on 27 May 2021).

2. Ostchega, Y.; Fryar, C.D.; Nwankwo, T.; Nguyen, D.T. Hypertension Prevalence Among Adults Aged 18 and Over: United States, 2017-2018. NCHS Data Brief, No 364; National Center for Health Statistics: Hyattsville, MD, USA, 2020.

3. Clark, C.E.; Smith, L.F.P.; Taylor, R.S.; Campbell, J.L. Nurse-led interventions to improve control of blood pressure in people with hypertension: Systematic review and meta-analysis. BMJ 2010, 341. [CrossRef]

4. Egan, B.M.; Li, J.; Hutchison, F.N.; Ferdinand, K.C. Hypertension in the United States, 1999 to 2012: Progress toward Healthy People 2020 goals. Circulation 2014, 130, 1692-1699. [CrossRef]

5. Chobanian, A.V.; Bakris, G.L.; Black, H.R.; Cushman, W.C.; Green, L.A.; Izzo, J.L., Jr.; Jones, D.W.; Materson, B.J.; Oparil, S.; Wright, J.T., Jr.; et al. Seventh report of the Joint National Committee on Prevention, Detection, Evaluation, and Treatment of High Blood Pressure. Hypertension 2003, 42, 1206-1252. [CrossRef] [PubMed]

6. Whelton, P.K.; Carey, R.M.; Aronow, W.S.; Casey, D.E.; Collins, K.J.; Dennison Himmelfarb, C.; DePalma, S.M.; Gidding, S.; Jamerson, K.A.; Jones, D.W.; et al. 2017 ACC/AHA/AAPA/ABC/ACPM/AGS/APhA/ASH/ASPC/NMA/PCNA Guideline for the Prevention, Detection, Evaluation, and Management of High Blood Pressure in Adults: Executive Summary: A Report of the American College of Cardiology / American Heart Association Task, F. Hypertension 2018, 71, 1269-1324. [CrossRef] [PubMed]

7. Williams, B.; Mancia, G.; Spiering, W.; Rosei, E.A.; Azizi, M.; Burnier, M.; Clement, D.L.; Coca, A.; de Simone, G.; Dominiczak, A.; et al. 2018 ESC/ESH Guidelines for the management of arterial hypertension. Eur. Heart J. 2018, 39, 3021-3104. [CrossRef] [PubMed]

8. Zhou, F.; Yu, T.; Du, R.; Fan, G.; Liu, Y.; Liu, Z.; Xiang, J.; Wang, Y.; Song, B.; Gu, X.; et al. Clinical course and risk factors for mortality of adult inpatients with COVID-19 in Wuhan, China: A retrospective cohort study. Lancet 2020, 395, 1054-1062. [CrossRef] 
9. Omboni, S.; McManus, R.J.; Bosworth, H.B.; Chappell, L.C.; Green, B.B.; Kario, K.; Logan, A.G.; Magid, D.J.; Mckinstry, B.; Margolis, K.L.; et al. Evidence and Recommendations on the Use of Telemedicine for the Management of Arterial Hypertension. Hypertension 2020, 76, 1368-1383. [CrossRef]

10. Wilkins, A. Local response in health emergencies: Key considerations for addressing the COVID-19 pandemic in informal urban settlements. Env. Urban. 2020, 32, 503-522. [CrossRef]

11. Sharma, A.; Ahmed, S.; Kaur, J.; Chawla, R.; Rejeeth, C. Exploring status of emergency drugs and vaccine development in Covid-19 pandemic: An update. VirusDisease 2021, 1-13. [CrossRef]

12. World Health Organization. WHO Coronavirus Disease (COVID-19) Dashboard. 2020. Available online: https:/ / covid19.who.int/ (accessed on 27 May 2021).

13. Bello, B.; Usehv, U. COVID-19: Are Non-Communicable Diseases Risk Factors for Its Severity? Am. J. Health Promot. 2021, 35, 720-729. [CrossRef]

14. Miner, H.; Fatehi, A.; Ring, D.; Reichenberg, J.S. Clinician Telemedicine Perceptions During the COVID-19 Pandemic. Telemed. e-Health 2021, 27. [CrossRef] [PubMed]

15. Ing, E.B.; Xu, Q.; Salimi, A.; Torun, N. Physician deaths from corona virus (COVID-19) disease. Occup. Med. 2020, 70, 370-374. [CrossRef]

16. Phillips, L.S.; Branch, W.T.; Cook, C.B.; Doyle, J.P.; El Kebbi, I.M.; Gallina, D.L.; Miller, C.D.; Zeimer, D.C.; Barnes, C.S. Clinical inertia. Ann. Intern. Med. 2001, 135, 825-834. [CrossRef]

17. Monahan, M.; Jowett, S.; Nickless, A.; Frassen, M.; Grant, S.; Greenfield, S.; Hobbs, F.D.R.; Hodgkinson, J.; Mant, J.; McManus, R. Cost-Effectiveness of Telemonitoring and Self-Monitoring of Blood Pressure for Antihypertensive Titration in Primary Care (TASMINH4). Hypertension 2019, 73, 1231-1239. [CrossRef] [PubMed]

18. Omboni, S.; Guarda, A. Impact of Home Blood Pressure Telemonitoring and Blood Pressure Control: A Meta-Analysis of Randomized Controlled Studies. Am. J. Hypertens. 2011, 24, 989-998. [CrossRef] [PubMed]

19. Filho, S.P.; Paffer, M.T.; Paffer, P.T.; Figeuiredo, M.C.; Veras, C.O.; Fonsêca, F.B. Home blood pressure monitoring: Report of a database of 1474 patients. J. Hypertens. 2021, 39, e121-e122. [CrossRef]

20. Clark, C.E.; Horvath, I.A.; Taylor, R.S.; Campbell, J.L. Doctors record higher blood pressures than nurses: Systematic review and meta-analysis. Br. J. Gen. Pract. 2014, 64, e223-e232. [CrossRef]

21. Cicolini, G.; Simonetti, V.; Comparcini, D.; Celiberti, I.; Nicola, M.D.; Capasso, L.M.; Flacco, M.E.; Bucci, M.; Mezzetti, A.; Manzoli, L. Efficacy of a nurse-led email reminder program for cardiovascular prevention risk reduction in hypertensive patients: A randomized controlled trial. Int J. Nurs. Stud. 2014, 51, 833-843. [CrossRef]

22. Mohsen, M.M.; Riad, N.A.; Badawy, A.E.; Abd El Gafar, S.E.; Abd El-Hammed, B.M.; Eltomy, E.M. Tele-nursing versus Routine Outpatient Teaching for Improving Arterial Blood Pressure and Body Mass Index for Hypertensive Patients. Am. J. Nurs. Res. 2020, 8, 18-26.

23. Hebert, P.; Sisk, J.E.; Tuzzio, L.; Casabianca, J.M.; Pogue, V.A.; Wang, J.J.; Chen, Y.; Cowles, C.; McLaughlin, M.A. Nurse-led disease management for hypertension control in a diverse urban community: A randomized trial. J. Gen. Intern. Med. 2012, 27, 630-639. [CrossRef] [PubMed]

24. Hill, M.N.; Bone, L.R.; Hilton, S.C.; Roary, M.C.; Kelen, G.D.; Levine, D.M. A clinical trial to improve high blood pressure care in young urban black men: Recruitment, follow-up, and outcomes. Am. J. Hypertens. 1999, 12, 548-554. [CrossRef]

25. McMahon, G.T.; Gomes, H.E.; Hickson Hohne, S.; Hu, T.M.; Levine, B.A.; Conlin, P.R. Web-based care management in patients with poorly controlled diabetes. Diabetes Care 2005, 28, 1624-1629. [CrossRef] [PubMed]

26. Artinian, N.T.; Flack, J.M.; Nordstrom, C.K.; Hockman, E.M.; Washington, O.G.M.; Jen, K.C.; Fathy, M. Effects of nurse-managed telemonitoring on blood pressure at 12-month follow-up among urban African Americans. Nurs. Res. 2007, 56, 312-322. [CrossRef]

27. Bosworth, H.B.; Olsen, M.K.; McCant, F.; Harrelson, M.; Gentry, P.; Rose, C.; Goldstein, M.K.; Hoffman, B.B.; Powers, B.; Oddone, E.Z. Hypertension Intervention Nurse Telemedicine Study (HINTS): Testing a multifactorial tailored behavioral/educational and a medication management intervention for blood pressure control. Am. Heart J. 2007, 153, 918-924.

28. Laurant, M.; Reeves, D.; Hermens, R.; Braspenning, J.; Grol, R.; Sibbald, B. Substitution of doctors by nurses in primary care. Cochrane Database Syst. Rev. 2005, CD001271. [CrossRef]

29. Fahey, T.; Schroeder, K.; Ebrahim, S.; Glynn, L. Interventions used to improve control of blood pressure in patients with hypertension. Cochrane Database Syst. Rev. 2010, CD005182. [CrossRef]

30. McManus, R.; Mant, J.; Franssen, M.; Nickless, A.; Schwartz, C.; Hodgkinson, J.; Bradburn, P.; Farmer, A.; Grant, S.; Greenfield, S.; et al. Telemonitoring and/or self-monitoring of blood pressure in hypertension (TASMINH4): A randomised controlled trial. J. Hypertens. 2018, 36, e5. [CrossRef]

31. Glynn, L.G.; Murphy, A.W.; Smith, S.M.; Schroeder, K.; Fahey, T. Self-monitoring and other non-pharmacological interventions to improve the management of hypertension in primary care: A systematic review. Br. J. Gen. Pract. 2010, 60, e476-e488. [CrossRef] [PubMed]

32. Omboni, S.; Gazzola, T.; Carabelli, G.; Parati, G. Clinical usefulness and cost effectiveness of home blood pressure telemonitoring: Meta-analysis of randomized controlled studies. J. Hypertens. 2013, 31, 455-468. [CrossRef]

33. Duan, Y.; Xie, Z.; Dong, F.; Wu, Z.; Lin, Z.; Sun, N.; Xu, J. Effectiveness of home blood pressure telemonitoring: A systematic review and meta-analysis of randomised controlled studies. J. Hum. Hypertens. 2017, 31, 427-437. [CrossRef] 
34. Cumpston, M.; Li, T.; Page, M.J.; Chandler, J.; Welch, V.A.; Higgins, J.P.; Thomas, J. Updated guidance for trusted systematic reviews: A new edition of the Cochrane Handbook for Systematic Reviews of Interventions. Cochrane Database Syst. Rev. 2019, 10, ED000142. [CrossRef]

35. Choi, W.S.; Kim, N.S.; Kim, A.Y.; Woo, H. A Systematic Review and Meta-Analysis of Nurse-Coordinated Blood Pressure Telemonitoring for Urban Hypertensive Patients During the Covid-19 Era. PROSPERO. 2020, p. CRD42020222789. Available online: https:/ / www.crd.york.ac.uk/PROSPERO/display_record.php?ID=CRD42020222789 (accessed on 30 April 2020).

36. Kerry, S.M.; Markus, H.S.; Khong, T.K.; Cloud, G.C.; Tulloch, J.; Coster, D.; Ibison, J.; Oakeshott, P. Home blood pressure monitoring with nurse-led telephone support among patients with hypertension and a history of stroke: A community-based randomized controlled trial. CMAJ 2013, 185, 23-31. [CrossRef]

37. Kim, K.B.; Han, H.R.; Huh, B.; Nguyen, T.; Lee, H.; Kim, M.T. The effect of a community-based self-help multimodal behavioral intervention in Korean American seniors with high blood pressure. Am. J. Hypertens. 2014, 27, 1199-1208. [CrossRef] [PubMed]

38. Pan, F.; Wu, H.; Liu, C.; Zhang, X.; Peng, W.; Wei, X.; Gao, W. Effects of home telemonitoring on the control of high blood pressure: A randomised control trial in the Fangzhuang Community Health Center, Beijing. Aust. J. Prim. Health 2018, 24, 398-403. [CrossRef] [PubMed]

39. Pour, R.E.; Aliyari, S.; Farsi, Z.; Ghelich, Y. Comparing the effects of interactive and noninteractive education using short message service on treatment adherence and blood pressure among patients with hypertension. Nurs. Midwifery Stud. 2020, 9, 68-76.

40. Zha, P.; Qureshi, R.; Porter, S.; Chao, Y.Y.; Pacquiao, D.; Chase, S.; O’Brien-Richardson, P. Utilizing a Mobile Health Intervention to Manage Hypertension in an Underserved Community. West. J. Nurs. Res. 2020, 42, 201-209. [CrossRef]

41. Borenstein, M.; Hedges, L.V.; Higgins, J.P.; Rothstein, H.R. Introduction to Meta-Analysis; John Wiley \& Sons: Hoboken, NJ, USA, 2011.

42. Review Manager (RevMan) [Computer program]. Version 5.4. The Cochrane Collaboration, 2020.

43. Higgins, J.P.; Thompson, S.G.; Deeks, J.J.; Altman, D.G. Measuring inconsistency in meta-analyses. BMJ 2003, 327, 557-560. [CrossRef] [PubMed]

44. Cohen, J. Statistical Power Analysis for the Behavioral Sciences, 2nd ed.; Lawrence Erlbaum Associates: Mahwah, NJ, USA, 1988.

45. Borenstein, M.; Hedges, L.; Higgins, J.; Rothstein, H. Comprehensive Meta-Analysis, Version 2; 104; Biostat: Englewood, NJ, USA, 2005.

46. Bosworth, H.B.; Powers, B.J.; Olsen, M.K.; McCant, F.; Grubber, J.; Smith, V.; Gentry, P.W.; Rose, C.; Van Houtven, C.; Wang, V.; et al. Home blood pressure management and improved blood pressure control: Results from a randomized controlled trial. Arch. Intern. Med. 2011, 171, 1173-1180. [CrossRef] [PubMed]

47. Park, M.; Kim, H.; Kim, K. Cellular phone and Internet-based individual intervention on blood pressure and obesity in obese patients with hypertension. Int. J. Med. Inform. 2009, 78, 704-710. [CrossRef] [PubMed]

48. Shea, S.; Weinstock, R.S.; Starren, J.; Teresi, J.; Palmas, W.; Field, L.; Morin, P.; Goland, R.; Izquierdo, R.E.; Wolff, T.; et al. A randomized trial comparing telemedicine case management with usual care in older, ethnically diverse, medically underserved patients with diabetes mellitus. J. Am. Med. Inform. Assoc. 2006, 13, 40-51. [CrossRef]

49. Wakefield, B.J.; Holman, J.E.; Ray, A.; Scherubel, M.; Adams, M.R.; Hillis, S.L.; Rosenthal, G.E. Effectiveness of home telehealth in comorbid diabetes and hypertension: A randomized, controlled trial. Telemed. e-Health 2011, 17, 254-261. [CrossRef]

50. Higgins, J.P.T.; Altman, D.G.; Sterne, J.A.C. Assessing risk of bias in included studies. In Cochrane Handbook for Systematic Reviews of Interventions. Version 5.1.0 (Updated March 2011); Higgins, J.P.T., Green, S., Eds.; The Cochrane Collaboration, John Wiley \& Sons: Chichester, UK, 2011; Available online: http/ / www.handbook.cochrane.org (accessed on 31 July 2019).

51. Duval, S.; Tweedie, R. Trim and fill: A simple funnel-plot-based method of testing and adjusting for publication bias in meta-analysis. Biometrics 2000, 56, 455-463. [CrossRef] [PubMed]

52. World Health Organization. A Health Telematics Policy in Support of WHO's Health-For-All Strategy for Global Health Development. Report of the WHO Group Consultation on Health Telematics, December 11-16, 1997; World Health Organization: Geneva, Switzerland, 1998.

53. Chu, D.K.; Akl, E.A.; Duda, S.; Solo, K.; Yaacoub, S.; Schünemann, H.J. Physical distancing, face masks, and eye protection to prevent person-to-person transmission of SARS-CoV-2 and COVID-19: A systematic review and meta-analysis. Lancet 2020, 395, 1973-1987. [CrossRef]

54. Blood Pressure Lowering Treatment Trialists' Collaboration. Blood pressure-lowering treatment based on cardiovascular risk: A meta-analysis of individual patient data. Lancet 2014, 384, 591-598. [CrossRef]

55. Choi, W.S.; Choi, J.H.; Oh, J.; Shin, I.; Yang, J. Effects of Remote Monitoring of Blood Pressure in Management of Urban Hypertensive Patients: A Systematic Review and Meta-Analysis. Telemed. e-Health 2020, 26, 744-759. [CrossRef] [PubMed]

56. Aas, I.H.M. Telemedical work and cooperation. J. Telemed. Telecare 2001, 7, 212-218. [CrossRef]

57. Jacob, C.; Sanchez-Vazquez, A.; Ivory, C. Social, Organizational, and Technological Factors Impacting Clinicians' Adoption of Mobile Health Tools: Systematic Literature Review. JMIR mHealth uHealth 2020, 8, e15935. Available online: https:/ / preprints. jmir.org/preprint/15935 (accessed on 10 April 2020). [CrossRef]

58. Palmas, W.; Teresi, J.; Morin, P.; Wolff, L.T.; Field, L.; Eimicke, J.P.; Capps, L.; Prigollini, A.; Orbe, I.; Weinstock, R.S.; et al. Recruitment and enrollment of rural and urban medically underserved elderly into a randomized trial of telemedicine case management for diabetes care. Telemed. e-Health 2006, 12, 601-607. [CrossRef] [PubMed]

59. Smith, A.C.; Thomas, E.; Snoswell, C.L.; Haydon, H.; Mehrotra, A.; Clemensen, J.; Caffery, L.J. Telehealth for global emergencies: Implications for coronavirus disease 2019 (COVID-19). J. Telemed. Telecare 2020, 26, 309-313. [CrossRef] 